\title{
A dichotomy for some elementarily generated modal logics
}

\author{
Stanislav Kikot
}

October 6, 2018

\begin{abstract}
In this paper we consider the normal modal logics of elementary classes defined by first-order formulas of the form $\forall x_{0} \exists x_{1} \ldots \exists x_{n} \bigwedge x_{i} R_{\lambda} x_{j}$. We prove that many properties of these logics, such as finite axiomatisability, elementarity, axiomatisability by a set of canonical formulas or by a single generalised Sahlqvist formula, together with modal definability of the initial formula, either simultaneously hold or simultaneously do not hold.
\end{abstract}

\section{Introduction}

This research was motivated by the following observation. Consider two first-order conditions: $\forall x \exists y(x R y \wedge$ $y R x)$ and $\forall x \exists y(x R y \wedge y R y)$ (see Figure 1). The first one is modally definable by a Sahlqvist formula $p \rightarrow \diamond \diamond p$ while the second is not, since it does not reflect ultrafilter extensions (e.g., [3], p. 142). The difference between these two formulas becomes even more palpable if we look at the modal logics $L_{1}$ and $L_{2}$ of the corresponding elementary classes. While $L_{1}$ is axiomatisable (with the standard rules of Substitution, Modus Ponens and Necessitation) by a single Sahlqvist formula, $L_{2}$ is not finitely axiomatisable and the class of Kripke frames $\left\{\mathfrak{F} \mid \mathfrak{F} \models L_{2}\right\}$ is not definable by any formula of first-order logic [16]. Moreover, any axiomatisation of $L_{2}$ requires infinitely many non-canonical formulas [15]. On the other hand, both formulas have a common structure and can be represented by graphs as in Figure 1, which are called diagrams in this paper. The author decided that this issue is worthy of additional explanation. So a study with the purpose of classifying all elementary classes $\mathcal{C}$ definable by formulas of the form $\forall x_{0} \mathrm{e}\left(x_{0}\right)$ where $\mathrm{e}\left(x_{0}\right)=\exists x_{1} \ldots \exists x_{n} \wedge x_{i} R_{\lambda} x_{j}$ according to the following model-theoretic properties (whose precise definitions will be given in Section 2) was undertaken:

$(I-i) \mathrm{e}\left(x_{0}\right)$ is modally definable by a generalised Sahlqvist formula;

(I-ii) $\mathrm{e}\left(x_{0}\right)$ is locally modally definable;

(I-iii) $\forall x_{0} \mathrm{e}\left(x_{0}\right)$ is globally modally definable;

(I-iv) $\log (\mathcal{C})$ is axiomatisable by a generalised Sahlqvist formula;

$(I-v) \log (\mathcal{C})$ is finitely axiomatisable;

$(I-v i) \log (\mathcal{C})$ is axiomatisable by a set of modal formulas containing finitely many propositional variables;

(I-vii) $\log (\mathcal{C})$ is axiomatisable by a set of canonical formulas;

(I-viii) $\log (\mathcal{C})$ is axiomatisable by a modal formula $\phi$ and a set of canonical formulas;

$(I-i x)\{\mathfrak{F} \mid \mathfrak{F} \models \log (\mathcal{C})\}=\mathcal{C}$;

$(I-x)\{\mathfrak{F} \mid \mathfrak{F} \models \log (\mathcal{C})\}$ is elementary ${ }^{1}$.

\footnotetext{
${ }^{1}$ In this paper we call a class of first-order models elementary if it is defined by a single first-order sentence, and $\Delta$-elementary if it is defined by a set of sentences.
} 


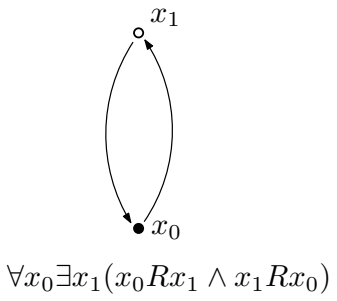

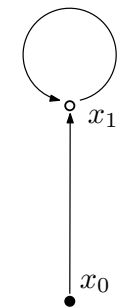

$\forall x_{0} \exists x_{1}\left(x_{0} R x_{1} \wedge x_{1} R x_{1}\right)$

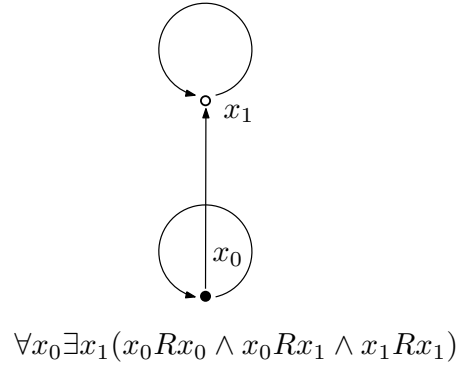

Figure 1: Formulas and their diagrams (universally quantified variables are black, existentially quantified variables are white)

Briefly, we prove that for any class $\mathcal{C}$ in question, conditions $(I-i)-(I-x)$ either simultaneously hold, or simultaneously do not hold, and this is determined by the existence in the corresponding diagram of an undirected cycle not passing through the universally quantified point, provided that the diagram is "minimal", i.e., none of its edges may be removed without affecting the corresponding formula, and "rooted", i.e., each of its points is reachable from $x_{0}$ via a directed path.

We exclude from our list such algorithmical properties as decidability, finite model property and complexity, and do not deal with them in this paper, since an easy (but seemingly unpublished) argument shows that all logics in our class have f.m.p. and are PSPACE-complete regardless of the mentioned cycle. But we cannot help mentioning that the dichotomies in the complexity-theoretic setting have recently become known to the logical community. For example, in [13] the modal logics given by universal Horn sentences are classified into those that are in NP and those that are PSPACE-hard and this classification was further refined in [27]. The authors of [25] classified universal relational constraints with respect to the complexity of reasoning in the description logic $\mathcal{E} \mathcal{L}$.

This work is in line with current research in theoretical modal logic. First, this result can be considered as a straighforward generalisation of Hughes' paper [16] about the reflexive-successor logic. The axiomatics of [16] was generalised in [1] to the case of first-order conditions of the form $\forall x \exists y\left(x R_{\lambda} y \wedge \phi(y)\right)$ where $\phi(y)$ is a generalised Kracht formula [18], and for some particular logics of this form finite axiomatisability, the finite model property and elementarity are studied there. The authors of [1] also conjectured that within their class there is a coincidence between finite axiomatisability and elementarity, and between $\Delta$-elementarity and elementarity (cf. [2]).

Another central problem of modal logic is: given an elementary class, i.e., a first-order formula, provide an explicit axiomatisation of the corresponding modal logic (this was done in [14]), and describe its properties, for example, in terms of $(I-i)-(I-x)$ (cf. problems 6.6 and 6.8 ibid.) Since the product of two elementary classes is elementary [9], the school of many dimensional modal logic deals mainly with such problems (e.g., [23],[24], and [8] for older results). In general, the algorithmic problem 'given a firstorder formula, decide whether each of $(I-i)-(I-x)$ holds' should be undecidable due to the undecidability of first-order logic. E.g., for (I-iii) it is Chagrova's theorem [7],[6], but it seems plausible that using the method of [6] one can prove such undecidability results for all items. On the other hand, when we restrict attention to a fragment of the first-order language with decidable implication, then we have chances to obtain such algorithmic criteria (as, e.g. in [19]), and the present paper is a step in this direction.

One more fundamental problem of modal logic is to study which implications between $(I-i)-(I-x)$ hold, and which of these conditions are independent. A brief summary of known results is given in [14] (see discussion after Problem 6.6), and we think that our result is interesting in this context.

This paper also concerns a phenomenon called "canonicity in the limit", referring to the logics (or, more generally, in terms of universal algebra, sets of equations that are true on some elementary class), that are canonical, but cannot be axiomatized by canonical formulas, and, even more, any axiomatisation of such logics requires infinitely many non-canonical axioms. Beside the aforementioned Hughes' logic, this issue includes the equational theories of representable relational [15] and cylindrical [4] algebras, and the well-known McKinsey-Lemmon logic [11]. This issuee was elaborated further in [5]. It turns out that all logics under consideration in the present paper excepting those which are generally Sahlqvist have this property; thus, what was thought pathological for elementarily generated modal logics can now be seen to be the norm. 

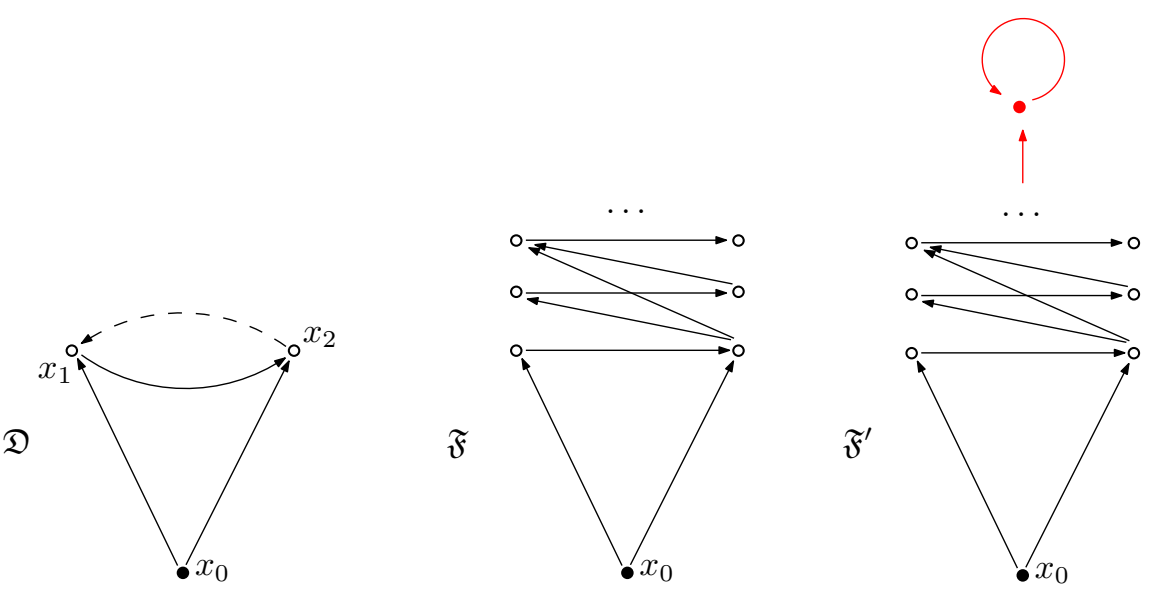

Figure 2:

And - last but not least - this paper can be regarded as a contribution to the question of whether there are natural generalisations of the Sahlqvist-Kracht correspondence in the basic modal language, besides [12], [28], [29], [18]. From our result it follows that the Kracht's theorem [21], [22] cannot be generalised further within the formulas of our class with any of $(I-i)-(I-x)$ as its consequence.

The outline of the paper is as follows. First, we take a diagram, all the cycles of which pass through the root, and use the result from [20] stating that the corresponding first-order formula e $\left(x_{0}\right)$ is modally definable by a generalised Sahlqvist formula, and so, by the generalised Sahlqvist theorem [12], (I-i) $(I-x)$ hold. Then we have to take a diagram with a cycle not passing through the root, and show that $(I-i)-(I-x)$ do not hold. This can be done only if e $\left(x_{0}\right)$ is "minimal", i.e., it does not contain atoms which can be thrown away without changing e $\left(x_{0}\right)$ semantically. Indeed, the diagram in Figure 1 on the right has a cycle not passing through the root, but it is modally definable, since it is equivalent to the reflexivity condition. So we additionally assume that the diagram is minimal. This can be done without any loss of generality, since we may take any formula of our class and remove superfluous edges until the formula becomes minimal. Under this assumption we prove that $(I-i)-(I-x)$ do not hold in Sections $4-8$. For this purpose we need the axiomatisations of the corresponding modal logics, constructed in Section 3. Then we construct 'non-standard frames' for our logic ensuring falsity of $(I-i)-(I-x)$.

To understand the intuition underlying these non-standard frames and the problems arising in their construction, let us turn to [20], where similar frames are used to prove that (I-ii) does not hold for any diagram with a cycle of the given form. Roughly, we temporarily remove one of the edges of a diagram $\mathfrak{D}$ belonging to a cycle (dashed edge in $\mathfrak{D}$ in Figure 2), then clone the rest of the diagram (more precisely, all points except the root) $\omega$ times, preserving edges of the diagram inside each layer and between the root and any layer, and insert the deleted arrow between corresponding points of different layers, from layers with lesser numbers to layers with greater numbers. Denote this Kripke frame by $\mathfrak{F}$ (Figure 2 , in the middle). One can show that $\mathfrak{F}$ has a root $r$ and satisfies $\mathfrak{F} \not=\mathrm{e}^{\mathfrak{D}}(r)$ and $(\mathfrak{F})^{\text {u.e. }}=\mathrm{e}^{\mathfrak{D}}(r)$, yielding the negation of $(I-i i)$. Now imagine that we want to generalise this construction to prove that (I-iii) does not hold. In this case we need our construction to validate a stronger condition $(\mathfrak{F})^{\text {u.e. }} \models \forall x \mathrm{e}^{\mathfrak{D}}(x)$, so the construction must be modified accordingly. In many cases we can just 'put on top' of $\mathfrak{F}$ a reflexive point (Figure 2, on the right), but in general this approach does not work and a more subtle construction is required. So in Lemma 4.1 we 'saturate' $\mathfrak{D}$ by adding new points until it satisfies $\forall x \mathrm{e}^{\mathfrak{D}}(x)$, and thus construct a Kripke frame $\mathfrak{F}_{+}^{\mathfrak{D}}$. Then we delete an edge of the cycle, obtaining $\mathfrak{F}_{-}^{\mathfrak{D}}$, and use $\mathfrak{F}_{+}^{\mathfrak{D}}$ and $\mathfrak{F}_{-}^{\mathfrak{D}}$ instead of $\mathfrak{D}$ in the construction of $\mathfrak{F}$. Another component of these non-standard frames are probabilistic graphs of I. Hodkinson and Y. Venema. 


\section{Preliminaries}

\subsection{Modal Formulas and Logics}

Fix a set of propositional variables $P V=\left\{p_{1}, p_{2}, \ldots\right\}$, a set of nominals $N V=\left\{j_{1}, j_{2}, \ldots\right\}$, and a set of indices $\Lambda$. Propositional variables are also denoted by $p, q, r, \ldots$ and nominals (only in this section) by $i$ and $j$. Hybrid formulas are built from propositional variables and nominals using the constant $\perp$, the binary connective $\rightarrow$, and unary connectives $\diamond_{\lambda}$ for $\lambda \in \Lambda$ and $\exists i$ for $i \in N V$. Other constructs are defined as usual: in particular, $\square_{\lambda}$ is a shorthand for $\neg \diamond_{\lambda} \neg$ and $\forall i$ is a shorthand for $\neg \exists i \neg$. A Kripke frame is a tuple $\mathfrak{F}=\left(W^{\mathfrak{F}},\left(R_{\lambda}^{\mathfrak{F}}: \lambda \in \Lambda\right)\right)$ where $W^{\mathfrak{F}}$ is a set (the carrier) and the $R_{\lambda}^{\mathfrak{F}}$ are binary relations on $W^{\mathfrak{F}}$; instead of $(x, y) \in R_{\lambda}^{\mathfrak{F}}$ we often write $x R_{\lambda}^{\mathfrak{F}} y$ or $\mathfrak{F}=x R_{\lambda} y$. We consider the components of $\mathfrak{F}$ as sets rather than the parts of the interpretation mapping, so occasionly their superscripts may be different from $\mathfrak{F}$ or just omitted. A map $\theta: P V \rightarrow \mathcal{P}\left(W^{\mathfrak{F}}\right)$ is called a propositional valuation (for a frame $\mathfrak{F}$ ), and a map $\tau: N V \rightarrow W^{\mathfrak{F}}$ is called a nominal valuation. A propositional valuation $\theta$ in a Kripke frame is called $k$-generated, if there are at most $k$ different propositional variables $p$, such that $\theta(p) \neq \emptyset$. Given a Kripke frame $\mathfrak{F}$ and valuations $\theta$ and $\tau$, we define the truth of hybrid formulas in a point $x \in W^{\mathfrak{F}}$ as usual:

$$
\begin{gathered}
\mathfrak{F}, \theta, \tau, x \models p \Longleftrightarrow x \in \theta(p) ; \quad \mathfrak{F}, \theta, \tau, x \models j \Longleftrightarrow x=\tau(j) ; \quad \mathfrak{F}, \theta, \tau, x \models \perp \text { never } ; \\
\mathfrak{F}, \theta, \tau, x \models \diamond_{\lambda} \phi \Longleftrightarrow \exists y \in W^{\mathfrak{F}} \text { such that } x R_{\lambda}^{\mathfrak{F}} y \text { and } \mathfrak{F}, \theta, \tau, y \models \phi ; \\
\mathfrak{F}, \theta, \tau, x \models \phi \rightarrow \psi \Longleftrightarrow \text { either } \mathfrak{F}, \theta, \tau, x \mid \models \phi \text { or } \mathfrak{F}, \theta, \tau, x \models \psi ; \\
\quad \text { for some nominal valuation } \tau^{\prime} \text { such that } \\
\mathfrak{F}, \theta, \tau, x \models \exists i \phi \Longleftrightarrow \quad \tau^{\prime}(j)=\tau(j) \text { for all } j \in N V \backslash\{i\} \text { we have } \mathfrak{F}, \theta, \tau^{\prime}, x \models \phi .
\end{gathered}
$$

A modal formula is a hybrid formula without nominals and nominal quantifiers. The truth of a modal formula $\phi$ at a point $w \in W^{\mathfrak{F}}$ of a Kripke frame $\mathfrak{F}$ depends only on the propositional valuation $\theta$ and is denoted by $\mathfrak{F}, \theta, w \models \phi$. A modal formula $\phi$ is said to be valid in a point $w \in W^{\mathfrak{F}}$ of a Kripke frame $\mathfrak{F}$ (denoted by $\mathfrak{F}, w \models \phi$ ) if for all valuations $\theta$ we have $\mathfrak{F}, \theta, w \models \phi$, and is said to be valid in a Kripke frame $\mathfrak{F}$ (denoted $\mathfrak{F} \models \phi$ ) if for all $w \in W^{\mathfrak{F}}$ we have $\mathfrak{F}, w=\phi$.

We can regard Kripke frames as models for the classical first-order language $\mathcal{L} f_{\Lambda}$, the signature of which consists of binary predicate symbols $R_{\lambda}$ for all $\lambda \in \Lambda$. We assume that $\mathcal{L} f_{\Lambda}$ contains equality. The formulas of this language are called in the sequel simply first-order formulas. Thus the truth relation $\mathfrak{F} \models \mathrm{a}$ is also defined for closed first-order formulas a, also called first-order sentences. A first-order sentence a is said to be globally modally definable if there exists a modal formula $\phi$ such that for any Kripke frame $\mathfrak{F}, \mathfrak{F}=\phi$ iff $\mathfrak{F} \models$ a. A first-order formula a $(x)$ with a single free variable $x$ is said to be locally modally definable if for any Kripke frame $\mathfrak{F}$ and any point $w$ in $\mathfrak{F}, \mathfrak{F}, w \models \phi$ iff $\mathfrak{F}=\mathrm{a}(w)$ for some modal formula $\phi$.

Each first-order sentence a defines the class of Kripke frames $\operatorname{Mod}(a)=\{\mathfrak{F} \mid \mathfrak{F} \models a\}$. A class of Kripke frames $\mathcal{C}$ is said to be elementary if it is of this form, and $\Delta$-elementary if $\mathcal{C}=\bigcap_{k=1}^{\infty} \operatorname{Mod}\left(\mathrm{a}_{k}\right)$ for some sequence of first-order formulas $\left\{a_{k}\right\}$. Given a class of Kripke frames $\mathcal{C}$, by $\log (\mathcal{C})$ we denote the set of all modal formulas valid in all frames of $\mathcal{C}$. A normal modal logic is a set of modal formulas containing all propositional tautologies, the formulas

$$
\mathrm{K}_{\lambda}: \square_{\lambda}(p \rightarrow q) \rightarrow\left(\square_{\lambda} p \rightarrow \square_{\lambda} q\right), \quad \lambda \in \Lambda,
$$

and closed under inference rules Modus Ponens, Uniform Substitution and Necessitation:

$$
\frac{\phi, \phi \rightarrow \psi}{\psi}, \quad \frac{\phi}{\phi[\psi / p]}, \quad \frac{\phi}{\square_{\lambda} \phi} .
$$

It is easy to see that $\log (\mathcal{C})$ is always a normal modal logic. A set of modal formulas $\Sigma$ axiomatises a normal modal logic $L$ if $L$ is the minimal (w.r.t. set-theoretic inclusion) normal modal logic containing $\Sigma$, and in this case we write $L=\mathrm{K}+\Sigma$. A modal logic is said to be finitely axiomatisable if it is axiomatised by some finite $\Sigma$, and axiomatisable using finitely many variables if it is axiomatised by some $\Sigma$ such that only a finite number of propositional variables occur in $\Sigma$. A modal formula $\phi$ is said to be canonical if it is valid in the canonical frame of the normal modal logic axiomatised by $\phi$. For a set $B$ we denote its powerset by $\mathcal{P}(B)$. 


\subsection{Minimal Diagrams and Kripke frames}

Consider an arbitrary relational structure $\mathfrak{F}=\left(W^{\mathfrak{F}},\left(R_{\lambda}^{\mathfrak{F}}: \lambda \in \Lambda\right)\right)$ where $R_{\lambda}^{\mathfrak{F}}$ are binary relations on $W^{\mathfrak{F}}$. For a binary relation $R$ by $\breve{R}$ we denote its converse $\{(x, y) \mid(y, x) \in R\}$. A sequence $z_{1} \lambda_{1} z_{2} \lambda_{2} \ldots \lambda_{h} z_{h+1}$ where for all $i z_{i} \in W^{\mathfrak{F}}, \lambda_{i} \in \Lambda$ and $\left(z_{i}, z_{i+1}\right) \in R_{\lambda_{i}}^{\mathfrak{F}}$ is called a directed path of length $h$ connecting $z_{1}$ to $z_{h+1}$ in $\mathfrak{F}$. To obtain the definition of an undirected path, we put $\Lambda^{ \pm}=\Lambda \cup\left\{\lambda^{-} \mid \lambda \in \Lambda\right\}$, assume that $R_{\lambda^{-}}^{\widetilde{F}}=\breve{R_{\lambda}^{\widetilde{F}}}$ and replace $\Lambda$ with $\Lambda^{ \pm}$in the definition of a directed path. An undirected cycle is an undirected path of positive length $h$ with $z_{1}=z_{h+1}$ and not containing a subsequence of the form $z \lambda y \lambda^{-} z$ and $z \lambda^{-} y \lambda z$. The distance from $y \in W^{\mathfrak{F}}$ and $z \in W^{\mathfrak{F}}$ in $\mathfrak{F}$ (denoted by $\operatorname{Dist}_{\mathfrak{F}}(y, z)$ ) is the length of the shortest directed path connecting $y$ to $z$ in $\mathfrak{F}$; if such path does not exist, we put $\operatorname{Dist}_{\mathfrak{F}}(y, z)=\infty$.

In this paper we deal with pointed Kripke frames, in other words, with tuples of the form $\mathfrak{D}=$ $\left(W^{\mathfrak{D}},\left(R_{\lambda}^{\mathfrak{D}}: \lambda \in \Lambda\right), x_{0}^{\mathfrak{D}}\right)$, where $R_{\lambda}^{\mathfrak{D}}$ are binary relations on $W^{\mathfrak{D}}$ and $x_{0}^{\mathfrak{D}} \in W^{\mathfrak{D}}$. A pointed Kripke frame $\mathfrak{D}$ is called rooted if for any point $y$ of $W^{\mathfrak{D}}$ there exists a directed path leading from $x_{0}^{\mathfrak{D}}$ to $y$. A diagram is a pointed rooted Kripke frame with a finite domain. An undirected cycle in a diagram $\mathfrak{D}$ is said to be inner if it does not contain the root $x_{0}^{\mathfrak{D}}$.

Assume that $W^{\mathfrak{D}}=\left\{x_{0}, x_{1}, \ldots, x_{n}\right\}$ and that $x_{0}^{\mathfrak{D}}=x_{0}$. The symbols $x_{i}$ will play a double role: first, they are the points of the diagram, second, they are first-order variables in the formulas defined below. We set

$$
\mathrm{k}^{\mathfrak{D}}\left(x_{0}, x_{1}, \ldots, x_{n}\right)=\bigwedge\left\{x_{i} R_{\lambda} x_{j} \mid i, j \leq n, x_{i} R_{\lambda}^{\mathfrak{D}} x_{j}\right\}
$$

and

$$
\mathrm{e}^{\mathfrak{D}}\left(x_{0}\right)=\exists x_{1} \ldots \exists x_{n} \mathrm{k}^{\mathfrak{D}}\left(x_{0}, x_{1}, \ldots, x_{n}\right) .
$$

We say that a diagram $\mathfrak{D}^{\prime}=\left(W^{\mathfrak{D}^{\prime}},\left(R_{\lambda}^{\mathfrak{D}^{\prime}}: \lambda \in \Lambda\right), x_{0}^{\mathfrak{D}^{\prime}}\right)$ is obtained from a diagram $\mathfrak{D}=\left(W^{\mathfrak{D}},\left(R_{\lambda}^{\mathfrak{D}}\right.\right.$ : $\left.\lambda \in \Lambda), x_{0}^{\mathfrak{D}}\right)$ by deleting the edge $x R_{\lambda_{0}} y$ and write $\mathfrak{D}^{\prime}=\mathfrak{D}-\left(x, y, \lambda_{0}\right)$ if $(x, y) \in R_{\lambda_{0}}^{\mathfrak{D}}, W^{\mathfrak{D}^{\prime}}=W^{\mathfrak{D}}$, $x_{0}^{\mathfrak{D}^{\prime}}=x_{0}^{\mathfrak{D}}, R_{\lambda_{0}}^{\mathfrak{O}^{\prime}}=R_{\lambda_{0}}^{\mathfrak{D}} \backslash\{(x, y)\}$ and for all $\lambda \neq \lambda_{0} R_{\lambda}^{\mathfrak{O}^{\prime}}=R_{\lambda}^{\mathfrak{D}}$. A diagram $\mathfrak{D}$ is called globally (locally) minimal if for any diagram $\mathfrak{D}^{\prime}$ obtained from $\mathfrak{D}$ by deleting an edge it is not true that $\vdash_{F O L} \forall x_{0} \mathrm{e}^{\mathfrak{D}^{\prime}}\left(x_{0}\right) \rightarrow$ $\forall x_{0} \mathrm{e}^{\mathfrak{D}}\left(x_{0}\right)$ (respectively, $\left.\vdash_{F O L} \forall x_{0}\left(\mathrm{e}^{\mathfrak{D}^{\prime}}\left(x_{0}\right) \rightarrow \mathrm{e}^{\mathfrak{D}}\left(x_{0}\right)\right)\right) ; F O L$ here means the classical first-order logic. Global minimality implies local minimality, but the converse fails in general. For instance, the diagram $\mathfrak{D}$ corresponding to the formula $\mathrm{e}^{\mathfrak{D}}\left(x_{0}\right)=\exists x_{1} \exists x_{2}\left(x_{0} R x_{1} \wedge x_{1} R x_{2}\right)$ is locally but not globally minimal.

\subsection{Ultrafilters, ultrafilter extension and ultraproducts}

A set $u \subseteq \mathcal{P}(W)$ is an ultrafilter over a set $W$ if, for all $X, Y \subseteq W$,

(u1) if $X, Y \in u$, then $X \cap Y \in u$;

(u2) if $X \in u$ and $X \subset Y$, then $Y \in u$;

(u3) $X \notin u$ iff $\bar{X} \in u$, where $\bar{X}$ denote the complement $W \backslash X$.

From the definition it follows that for any ultrafilter $u$ over a set $W \emptyset \notin u$ and $W \in u$.

Given a frame $\mathfrak{F}=\left(W,\left(R_{\lambda}: \lambda \in \Lambda\right)\right)$, its ultrafilter extension is defined as the frame $\mathfrak{F}^{\text {u.e. }}=$ $\left(W^{\text {u.e. }},\left(R_{\lambda}^{u . e .}: \lambda \in \Lambda\right)\right.$ ), where $W^{\text {u.e. }}$ is the set of all ultrafilters over $W$, and $u R_{\lambda}^{u . e .} u^{\prime}$ holds for ultrafilters $u$ and $u^{\prime}$ iff $R_{\lambda}^{-1}(X) \in u$ for all $X \in u^{\prime}$, where $R_{\lambda}^{-1}(X)=\left\{z \mid z R_{\lambda} x\right.$ for some $\left.x \in X\right\}$. Given a point $a \in W$, the set $\pi_{a}=\{X \subseteq W \mid a \in X\}$ is obviously an ultrafilter; it is called the principal ultrafilter generated by $a$.

Lemma 2.1 ([3], p. 95). For any points $a, b$ in any frame $\mathfrak{F}, a R_{\lambda} b \Longleftrightarrow \pi_{a} R_{\lambda}^{u \cdot e \cdot} \pi_{b}$.

Lemma 2.2 ([3], p. 142). For any frame $\mathfrak{F}$ and modal formula $\phi, \mathfrak{F}^{\text {u.e. }} \models \phi$ implies $\mathfrak{F} \models \phi$.

Lemma 2.3. Let $u$ be an ultrafilter over $W, W=W_{1} \cup W_{2} \cup \cdots \cup W_{n}$, and $W_{i} \cap W_{j}=\emptyset$ for all $1 \leq i \neq j \leq n$. Then there exists a unique $i$ such that $W_{i} \in u$.

Proof. Induction on $n$. The cases $n=1, n=2$ follow immediately from the definition of an ultrafilter. Now suppose that the lemma is proven for some fixed $n$ and let us prove that it is true for $n+1$. Let $V_{1}=W_{1}, \ldots, V_{n-1}=W_{n-1}, V_{n}=W_{n} \cup W_{n+1}$. By inductive assumption we get either $W_{i} \in u$ for some $1 \leq i \leq n-1$, or $W_{n} \cup W_{n+1} \in u$. In the last case, if $W_{n} \notin u$ and $W_{n+1} \notin u$, then by (u3) we have 
$W_{1} \cup \cdots \cup W_{n-1} \cup W_{n+1} \in u$ and $W_{1} \cup \cdots \cup W_{n} \in u$, therefore, by (u2) $W_{1} \cup \cdots \cup W_{n-1} \in u$. This contradicts (u3) and $W_{n} \cup W_{n+1} \in u$. Thus there exists $1 \leq i \leq n+1$ such that $W_{i} \in u$. If there are two such $i$ 's, then $\emptyset \in u$.

We need yet another model-theoretic construction involving ultrafilters. Suppose that we have a family of Kripke frames $\mathfrak{F}^{i}=\left(W^{i},\left(R_{\lambda}^{i}: \lambda \in \Lambda\right)\right)$ for all $i \in \omega$ and a non-principal ultrafilter $u$ over $\omega$. We say that two sequences $\bar{\alpha}=\left(\alpha_{0}, \alpha_{1}, \alpha_{2}, \ldots\right)$ and $\bar{\beta}=\left(\beta_{0}, \beta_{1}, \beta_{2}, \ldots\right)$, where $\alpha_{i}, \beta_{i} \in W^{i}$ for all $i \in \omega$ are $u$-equivalent (denoted by $\bar{\alpha} \sim_{u} \bar{\beta}$ ), if $\left\{i \mid \alpha_{i}=\beta_{i}\right\} \in u$. By $W$ we denote the set of all such sequences. The equivalence class of a sequence $\alpha$ we denote by $\lceil\alpha\rceil$. The $\mathcal{L} f_{\Lambda}$-structure $\mathfrak{F}=\left(W^{\prime},\left(R_{\lambda}^{\prime}: \lambda \in \Lambda\right)\right)$, where

$$
W^{\prime}=\{\text { all sequences of points from } W\} / \sim_{u},
$$

and $\lceil\bar{\alpha}\rceil R_{\lambda}^{\prime}\lceil\bar{\beta}\rceil \Longleftrightarrow\left\{i \mid \alpha_{i} R_{\lambda}^{i} \beta_{i}\right\} \in u$ is called an ultraproduct of $\mathfrak{F}^{i}$ and is denoted by $\prod_{i \in \omega}^{u} \mathfrak{F}^{i}$.

Proposition 2.4 ([17], Thm 4.1.9). If $\mathcal{C}$ is an elementary class and $\left\{\mathfrak{F}^{i}\right\}$ is a sequence of Kripke frames from $\mathcal{C}$, then for any ultrafilter $u$ on $\omega, \prod_{i \in \omega}^{u} \mathfrak{F}^{i} \in \mathcal{C}$.

\subsection{Inverse limit of descriptive frames}

A general frame is a triple $\left(W,\left(R_{\lambda}: \lambda \in \Lambda\right), P\right)$ where $\left(W,\left(R_{\lambda}: \lambda \in \Lambda\right)\right)$ is a Kripke frame and $P \subseteq \mathcal{P}(W)$ is non-empty and closed under intersection, complement and $R_{\lambda}^{-1}$. A general frame $\left(W,\left(R_{\lambda}: \lambda \in \Lambda\right), P\right)$ is said to be a descriptive frame if

1. If $x, y \in W$ are distinct, then there is some $S \in P$ with $x \in S$ and $y \notin S$.

2. If $x, y \in W$ and $\neg R_{\lambda}(x, y)$, then there is some $S \in P$ with $x \in R_{\lambda}^{-1}(S)$ and $y \notin S$.

3. $\bigcap \mu \neq 0$ for every $\mu \subseteq P$ with finite intersection property.

Below we denote general frames by calligraphic letters to distinguish between them and Kripke frames. If $\mathfrak{F}=\left(W,\left(R_{\lambda}: \lambda \in \Lambda\right)\right)$ is a Kripke frame, we write $\mathfrak{F}^{+}$for $\left(W,\left(R_{\lambda}: \lambda \in \Lambda\right), \mathcal{P}(W)\right)$. Clearly, if $\mathfrak{F}$ is finite (i.e., $W$ is finite), then $\mathfrak{F}^{+}$is a descriptive frame. If $\mathcal{F}=\left(W,\left(R_{\lambda}: \lambda \in \Lambda\right), P\right)$ is a descriptive frame, we write $\mathcal{F}_{+}$for its underlying Kripke frame $\mathfrak{F}=\left(W,\left(R_{\lambda}: \lambda \in \Lambda\right)\right)$. Let $\mathcal{F}=\left(W,\left(R_{\lambda}: \lambda \in \Lambda\right), P\right)$ be a general frame and $\phi$ a modal formula. We say that $\phi$ is valid in $\mathcal{F}$, written $\mathcal{F}=\phi$ if $\left(W,\left(R_{\lambda}: \lambda \in\right.\right.$ $\Lambda), \theta, w \models \phi$ for every assigment $\theta: P V \rightarrow P$ and every $w \in W$.

An inverse family of descriptive frames is an object $\mathcal{I}=\left((I, \leq), \mathcal{F}_{i},\left(f_{i j}: i \geq j\right.\right.$ in $\left.\left.I\right)\right)$ where $(I, \leq)$ is an upwards-directed partial order ('upwards-directed' means that any finite subset of $I$ has an upper bound in $I), \mathcal{F}_{i}=\left(W_{i},\left(\left(R_{i}\right)_{\lambda}: \lambda \in \Lambda\right), P_{i}\right)$ is a descriptive frame for each $i \in I$, and for each $i, j \in I$ with $i \geq j f_{i j}: \mathcal{F}_{i} \rightarrow \mathcal{F}_{j}$ is a frame homomorphism such that (a) $f_{i i}$ is the identity map on $W_{i}$, and (b) $f_{j k} \circ f_{i j}=f_{i k}$ whenever $k \leq j \leq i$ in $I$.

The inverse limit $\lim _{\leftarrow} \mathcal{I}$ of $\mathcal{I}$ is defined to be $\mathcal{F}=\left(W,\left(R_{\lambda}: \lambda \in \Lambda\right), P\right)$ where

$$
\begin{gathered}
W=\left\{x \in \prod_{i \in I} W_{i}: f_{i j}\left(x_{i}\right)=x_{j} \text { for each } i \geq j \text { in } I\right\}, \\
R_{\lambda}=\left\{(x, y) \in W: x_{i}\left(R_{i}\right)_{\lambda} y_{i} \text { for each } i \in I\right\}, \\
P \text { is generated by }\left\{f_{i}^{-1}[S]: i \in I, S \in P_{i}\right\},
\end{gathered}
$$

where in the last line for each $i \in I f_{i}: W \rightarrow W_{i}$ is the projection given by $f_{i}(x)=x_{i}$.

Proposition 2.5 ([10], 1.1.2(8), 1.11.4). The inverse limit $\mathcal{F}$ of $\mathcal{I}$ is itself a descriptive frame. Moreover, for any modal formula $\phi$, if $\phi$ is valid in $F_{i}$ for each $i$, then $\phi$ is valid in $\mathcal{F}$.

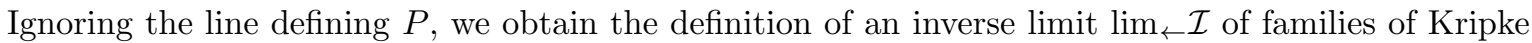
frames and graphs. If $I=(\omega, \leq)$ and $f_{i j}$ are clear from context, we denote the system simply $\left\{F_{i}\right\}$, and the inverse limit by $\lim _{\leftarrow} F_{i}$.

We regard a general frame $\left(W,\left(R_{\lambda}: \lambda \in \Lambda\right), P\right)$ as a first-order structure whose domain is the disjoint union of $W$ and $P$, with unary relations defining $W$ and $P$ and binary relations $R_{\lambda} \subseteq W \times W$ and $\epsilon \subseteq W \times P$ interpreted in the natural way. It is easy to write down a finite set $\Delta$ of first-order sentences expressing that a structure for this signature is a general frame. 
As is well known, every modal formula $\phi$ has a standard translation to a formula $S T_{x}(\phi)$ of firstorder logic with a free variable $x$. We modify this here by regarding propositional variables as first-order variables. For a propositional variable p, we define $S T_{x}(p)$ to be $x \epsilon p$. We put $S T_{x}(\top)=\top, S T_{x}(\phi \wedge \psi)$ and similarly for negation, $S T_{x}\left(\square_{\lambda} \phi\right)=\forall y\left(R_{\lambda}(x, y) \rightarrow S T_{y}(\phi)\right)$ and $S T_{x}\left(\diamond_{\lambda} \phi\right)=\exists y\left(R_{\lambda}(x, y) \wedge S T_{y}(\phi)\right)$, where $y$ is a new variable. For a formula $\phi\left(p_{1}, \ldots, p_{n}\right)$, we write $S T(\phi)$ for the universal closure $\forall x \in$ $W \forall p_{1} \ldots p_{n} \in P S T_{x}(\phi)$. For a set $X$ of modal formulas we write $S T(X)$ for $\{S T(\phi): \phi \in X\}$. Clearly, a modal formula $\phi$ is valid in a general frame $\mathcal{G}$ iff $S T(\phi)$ is true in it in first-order semantics:

$$
\mathcal{G} \models \phi \Longleftrightarrow \mathcal{G} \models S T(\phi) .
$$

Hence, $\phi$ is valid in a Kripke frame $\mathfrak{F}$ iff $S T(\phi)$ is true in $\mathfrak{F}^{+}$in first-order semantics:

$$
\mathfrak{F} \models \phi \Longleftrightarrow \mathfrak{F}^{+} \models S T(\phi) .
$$

Lemma 2.6 (Lemma 4.2 from [11]). Let $\mathcal{F}=\left(W,\left(R_{\lambda}: \lambda \in \Lambda\right), P\right)$ be a descriptive frame with countable $P$, and $\phi$ be a canonical formula. Then $\mathcal{F} \models \phi$ implies $\mathcal{F}_{+} \models \phi$.

The following lemma generalises the argument of (and is inspired by) Theorem 4.4 from [11], but seemingly does not follow from that paper.

Lemma 2.7. Let $\gamma_{i}$ be a sequence of modal formulas such that $\gamma_{i_{1}}$ implies $\gamma_{i_{2}}$ if $i_{2}<i_{1}$. Suppose that for all $l$ there exists $n$ such that for all $k$ there exists an inverse system of finite Kripke frames $\left\{\mathfrak{F}_{i}\right\}$ such that:

(L1) for all $i \mathfrak{F}_{i} \models \gamma_{k}$,

(L2) $\lim _{\leftarrow} \mathfrak{F}_{i} \models \gamma_{l}$,

(L3) $\lim _{\leftarrow} \mathfrak{F}_{i} \not \models \gamma_{n}$.

Then any axiomatisation of $L=\mathrm{K}+\left\{\gamma_{n}: n \in \omega\right\}$ has infinitely many non-canonical axioms.

Proof. Suppose on the contrary that $L$ is axiomatised by a single axiom $B$ together with a set $\Sigma$ of canonical formulas. Since $\Sigma \cup\{B\}$ and $\left\{\gamma_{k}: k<\omega\right\}$ axiomatise the same logic, the two first-order theories $\Delta \cup S T(\Sigma \cup\{B\})$ and $\Delta \cup\left\{S T\left(\gamma_{k}\right): k<\omega\right\}$ have the same models. Then by the first-order compactness we conclude:

(a) there is $l<\omega$ such that $\Delta \cup S T\left(\gamma_{l}\right) \models S T(B)$,

since $l$ is fixed, we fix $n$ from the condition of lemma, then

(b) there is a finite subset $X \subseteq \Sigma$ such that $\Delta \cup S T(X \cup\{B\}) \models S T\left(\gamma_{n}\right)$

(c) there is a finite $k$ such that $\Delta \cup S T\left(\gamma_{k}\right) \models S T(X)$, without loss of generality, we may take $k>l$.

The condition of the lemma gives us an inverse system $\left\{\mathfrak{F}_{i}\right\}$. Let $\mathcal{F}=\left(W,\left(R_{\lambda}: \lambda \in \Lambda\right), P\right)=$ $\lim _{\leftarrow}\left(\mathfrak{F}_{i}^{+}\right)$. Clearly, $\mathcal{F}_{+}=\lim _{\leftarrow} \mathfrak{F}_{i}$. By Proposition 2.5, since all $\mathfrak{F}_{i} \models \gamma_{k}, \mathcal{F} \models \gamma_{k}$. Plainly, $\mathcal{F} \models \Delta$. Now, by (c) and (I) we obtain that $\mathcal{F}=X$. The formulas in $X$ are assumed canonical, and $P$ by construction is countable, therefore by Lemma $2.6 \mathcal{F}_{+} \models X$ as well. By (II), $\left(\mathcal{F}_{+}\right)^{+} \models S T(X)$.

As $\mathcal{F}_{+}=\gamma_{l}$, (II) gives $\left(\mathcal{F}_{+}\right)^{+} \models S T\left(\gamma_{l}\right)$. Clearly, $\left(\mathcal{F}_{+}\right)^{+} \models \Delta$. So by $($ a $),\left(\mathcal{F}_{+}\right)^{+}=S T(B)$. Now we have $\left(\mathcal{F}_{+}\right)^{+} \models \Delta \cup S T(X \cup\{B\})$, so by (b) and (II) we arrive at $\mathcal{F}_{+} \models \gamma_{n}$, a contradition to (L3).

\section{Axiomatisation}

Fix a rooted diagram $\mathfrak{D}$. An axiomatisation of its normal modal $\operatorname{logic} L^{\mathfrak{P}}=\log \left(\forall x_{0} \mathrm{e}^{\mathfrak{P}}\left(x_{0}\right)\right)$ can be obtained using the algorithm from [14]. It allows one to write modal axioms for any normal modal logic generated by a first-order formula $\phi$ preserved under p-morphic images, disjoint unions and generated submodels, and this is our case. This algorithm is followed quite liberally, since we act within a very restricted class of formulas, and this allows us to keep the presentation simpler and closer to the ultimate goal of this paper. We also give an independent and 'handmade' proof of the soundness and completeness and invite connoisseurs to compare it with the general machinery from the quoted paper. They will 
definitely note that in terms of [14] the set $\Psi$ below is nothing else but a display and $\gamma_{\Psi}^{\mathfrak{P}}$ an approximant for the hybrid formula $\eta^{\mathfrak{D}}$. We also note that our axioms and completeness proof are similar to those in [30] for the "reflexive successor" logic.

We proceed in two stages: first, we construct a 'hybrid equivalent' of $\phi$, second, we translate these hybrid formulas into modal axioms. To translate $\mathrm{e}^{\mathfrak{D}}\left(x_{0}\right)$ into hybrid language, we need to construct a spanning tree ${ }^{2}$ for $\mathfrak{D}$.

Definition 3.1. A tuple $\mathfrak{T}=\left(W,\left(R_{\lambda}: \lambda \in \Lambda\right), r\right)$ is called a tree with a root $r$ if the following holds

1) $r \in W$

2) $R_{\lambda}^{-1}(r)=\emptyset$ for all $\lambda \in \Lambda$, where $R_{\lambda}^{-1}(x)=\left\{z \mid(z, x) \in R_{\lambda}\right\}$,

3) for all $x \in W \backslash\{r\}$ there is a unique directed path from $r$ to $x$.

A tuple $\mathfrak{T}=\left(W^{\mathfrak{D}},\left(R_{\lambda}^{\mathfrak{T}}: \lambda \in \Lambda\right), x_{0}\right)$ is called a spanning tree for a diagram $\mathfrak{D}=\left(W^{\mathfrak{D}},\left(R_{\lambda}^{\mathfrak{D}}: \lambda \in \Lambda\right), x_{0}\right)$, if $\mathfrak{T}$ is a tree, and for all $\lambda \in \Lambda, x, y \in W^{\mathfrak{D}} x R_{\lambda}^{\mathfrak{T}} y$ implies $x R_{\lambda}^{\mathfrak{D}} y$.

Proposition 3.2 (e.g., Lemma 5.5 from [20]). For any rooted diagram $\mathfrak{D}$ there exists a spanning tree $\mathfrak{T}$ for $\mathfrak{D}$.

Now, to every $x_{i}$ we assign a nominal $j_{i}$ and the hybrid formula

$$
\chi_{i}^{\mathfrak{P}}=j_{i} \wedge \bigwedge_{x_{i} R_{\lambda}^{\mathfrak{P} x_{k}}} \diamond_{\lambda} j_{k}
$$

Then, working by induction on $\mathfrak{T}$, moving from leaves to the root, to any point $x_{i}$ of $W^{\mathfrak{D}}$ we assign a hybrid formula

$$
\eta_{i}^{\mathfrak{D}}=\chi_{i}^{\mathfrak{D}} \wedge \bigwedge_{x_{i} R_{\lambda}^{\mathfrak{T}} x_{k}} \diamond_{\lambda} \eta_{k}^{\mathfrak{D}}
$$

Put $\eta^{\mathfrak{D}}=\eta_{0}^{\mathfrak{D}}$. Now note that $\mathrm{e}^{\mathfrak{D}}\left(x_{0}\right)$ is equivalent to $\exists j_{1} \ldots \exists j_{n} \eta^{\mathfrak{D}}$ in that sense that for any pointed Kripke frame $\mathfrak{F}$ and its point $w$ we have $\mathfrak{F}, w \models \eta^{\mathfrak{D}}$ iff $\mathfrak{F}=\mathrm{e}^{\mathfrak{D}}(w)$. By $\eta^{\mathfrak{D}}\left(\phi_{0}, \phi_{1}, \ldots, \phi_{n}\right)$ we denote the result of the substitution of modal formulas $\phi_{l}$ for nominals $j_{l}$ in the formula $\eta^{\mathfrak{D}}$ for $0 \leq l \leq n$. For a set of formulas $\Psi$ and a map $\kappa:\{0,1, \ldots, n\} \rightarrow \Psi$ let $\eta^{\mathfrak{D}}(\kappa)=\eta^{\mathfrak{D}}(\kappa(0), \kappa(1), \ldots, \kappa(n))$. Then we set

$$
\gamma_{\Psi}^{\mathfrak{D}}=\bigvee_{\kappa:\{0,1, \ldots, n\} \rightarrow \Psi} \eta^{\mathfrak{D}}(\kappa)
$$

where the disjunction is taken over all possible maps $\kappa:\{0,1, \ldots, n\} \rightarrow \Psi$. Finally, let $\Psi_{h}=\left\{\bar{p}^{\varepsilon} \mid \bar{p}=\right.$ $\left.\left\{p_{1}, \ldots, p_{h}\right\}, \varepsilon \in\{0,1\}^{h}\right\}$, where $h<\omega$ and $\bar{p}^{\varepsilon_{1} \ldots \varepsilon_{h}}=p_{1}^{\varepsilon_{1}} \wedge \cdots \wedge p_{h}^{\varepsilon_{h}}, p^{1}=p, p^{0}=\neg p$.

Theorem 3.3. $L^{\mathfrak{D}}$ is axiomatised by the set of formulas $\left\{\gamma_{\Psi_{h}}^{\mathfrak{D}} \mid h \in \omega\right\}$.

Proof. Soundness. Given a Kripke frame $\mathfrak{F}=\left(W^{\mathfrak{F}},\left(R_{\lambda}^{\mathfrak{F}}: \lambda \in \Lambda\right)\right)$, we show that for any $h \in \omega$ and $w_{0} \in W^{\mathfrak{F}}, \mathfrak{F} \models \mathrm{e}^{\mathfrak{D}}\left(w_{0}\right)$ implies $F, w_{0} \models \gamma_{\Psi_{h}}^{\mathfrak{P}}$. Suppose that $\mathfrak{F} \models \mathrm{e}^{\mathfrak{D}}\left(w_{0}\right)$, hence there exist points $w_{1}, \ldots w_{n} \in W^{\mathfrak{F}}$ such that $\mathfrak{F} \models \mathrm{k}^{\mathfrak{D}}\left(w_{0}, w_{1}, \ldots, w_{n}\right)$. Let $\theta$ be a valuation on $\mathfrak{F}$. For a fixed $i, 0 \leq i \leq n$, let $\varepsilon(i)$ be the boolean vector, the $j$-th component of which tells whether $w_{i}$ belongs to $\theta\left(p_{j}\right)$, and let $\kappa(i)=\bar{p}^{\varepsilon(i)}$. It is easy to check that $\mathfrak{F}, \theta, w_{0} \models \eta^{\mathfrak{D}}(\kappa)$.

Completeness. Let $\mathfrak{F}=\left(W^{\mathfrak{F}},\left(R_{\lambda}^{\mathfrak{F}}: \lambda \in \Lambda\right)\right)$ be the canonical frame for $\mathrm{K}+\left\{\gamma_{\Psi_{h}}^{\mathfrak{D}} \mid h \in \omega\right\}$, i.e., $W^{\mathfrak{F}}$ is the set of all maximal $\left(\mathrm{K}+\left\{\gamma_{\Psi_{h}}^{\mathfrak{D}} \mid h \in \omega\right\}\right)$-consistent sets (mcs) of formulas, and for any $w, w^{\prime} \in W^{\mathfrak{F}}$, $w R_{\lambda}^{\mathfrak{F}} w^{\prime}$ iff for all formulas $\phi \in w^{\prime} \diamond_{\lambda} \phi \in w$. We show that $\mathfrak{F} \models \forall x_{0} \mathrm{e}^{\mathfrak{D}}\left(x_{0}\right)$, and it follows that the logic $\mathrm{K}+\left\{\gamma_{\Psi_{h}}^{\mathbb{D}} \mid h \in \omega\right\}$ is Kripke complete with respect to the elementary class defined by this formula, i.e., $\forall x_{0} \mathrm{e}^{\mathfrak{D}}\left(x_{0}\right)$.

Take $w_{0} \in W^{\mathfrak{F}}$. Let us prove that there exist $w_{1}, \ldots, w_{n} \in W^{\mathfrak{F}}$ such that $\mathfrak{F}=\mathbf{k}^{\mathfrak{D}}\left(w_{0}, \ldots, w_{n}\right)$. By $\mathbf{V}$ we denote the set of word tuples $\overrightarrow{\mathbf{v}}=\left(\mathbf{v}_{0}, \mathbf{v}_{1}, \ldots, \mathbf{v}_{n}\right)$, where $\mathbf{v}_{i} \in\{0,1\}^{*}$. We set $\overrightarrow{\mathbf{v}} \prec \overrightarrow{\mathbf{u}}$ iff for every $0 \leq i \leq n \mathbf{v}_{i}$ is an initial segment of $\mathbf{u}_{i}$; thus $(\mathbf{V}, \prec)$ is a transitive tree with branching $2^{n+1}$ in each node.

\footnotetext{
${ }^{2}$ Traditionally, spanning trees are defined for unoriented graphs and are unoriented. Here we use an oriented modification of this notion, but we still call it a spanning tree.
} 
Suppose that $\psi_{1}, \psi_{2}, \psi_{3}, \ldots$ is an enumeration of all modal formulas. For a word $\mathbf{v} \in\{0,1\}^{*}$, by $\mathbf{v}^{j}$ we denote the $j$-th symbol of $\mathbf{v}$ and we set

$$
\mathbf{v}^{\#}=\bigwedge_{j=1}^{|\mathbf{v}|} \psi_{j}^{\mathbf{v}^{j}},
$$

where for a modal formula $\psi, \psi^{1}=\psi, \psi^{0}=\neg \psi$. By $\overrightarrow{\mathbf{v}}^{\#}$ we denote $\eta^{\mathfrak{D}}\left(\mathbf{v}_{0}^{\#}, \mathbf{v}_{1}^{\#}, \ldots, \mathbf{v}_{n}^{\#}\right)$. We say that $\overrightarrow{\mathbf{v}}$ is good if $\mathfrak{F}, \theta, w_{0} \models \overrightarrow{\mathbf{v}}^{\#}$, where $\theta$ is the canonical valuation.

Claim 1. If $\overrightarrow{\mathbf{v}} \prec \overrightarrow{\mathbf{u}}$ and $\overrightarrow{\mathbf{u}}$ is good, then so is $\overrightarrow{\mathbf{v}}$. Indeed, if $\overrightarrow{\mathbf{v}} \prec \overrightarrow{\mathbf{u}}$, then $\overrightarrow{\mathbf{u}}^{\#}$ implies $\overrightarrow{\mathbf{v}} \#$.

Claim 2. For each $m$ there is a good $\overrightarrow{\mathbf{v}}$ such that for all $1 \leq i \leq n\left|\mathbf{v}_{i}\right|=m$. To prove this claim, it is enough to notice that

$$
\bigvee_{\overrightarrow{\mathbf{v}}:|\mathbf{v} i|=m} \overrightarrow{\mathbf{v}}^{\#}
$$

is a substitution instance of $\gamma_{\Psi_{m}}^{\mathfrak{P}}$.

By König's lemma applied to $(\mathbf{V}, \prec)$ there exists a tuple of infinite strings

$$
\mathbf{v}^{\infty}=\left(\mathbf{v}_{0}^{\infty}, \mathbf{v}_{1}^{\infty}, \ldots, \mathbf{v}_{n}^{\infty}\right)
$$

such that any tuple formed by the initial segments of its components is good. By setting $w_{i}=\left\{\psi_{j} \mid\right.$ $\left.\left(\mathbf{v}_{i}^{\infty}\right)^{j}=1\right\} \cup\left\{\neg \psi_{j} \mid\left(\mathbf{v}_{i}^{\infty}\right)^{j}=0\right\}$ for $1 \leq i \leq n$, the tuple $\overrightarrow{\mathbf{v}}^{\infty}$ gives rise to mcs'es $w_{1}, \ldots, w_{n}$. It is clear that $w_{0}=\left\{\psi_{j} \mid\left(\mathbf{v}_{0}^{\infty}\right)^{j}=1\right\} \cup\left\{\neg \psi_{j} \mid\left(\mathbf{v}_{0}^{\infty}\right)^{j}=0\right\}$.

Now, we take $h, l$ and $\lambda$ such that $x_{h} R_{\lambda}^{\mathfrak{D}} x_{l}$ and prove that $w_{h} R_{\lambda}^{\mathfrak{F}} w_{l}$ in the canonical model. Suppose that for some $i \psi_{i} \in w_{l}$ but $\diamond \psi_{i} \notin w_{h}$. But $\square_{\lambda} \neg \psi_{i}=\psi_{k}$ for some $k$. Take $m=\max (i, k)$, and by $\overrightarrow{\mathbf{v}}$ denote the word vector formed by the first $m$ bits of components of $\overrightarrow{\mathbf{v}}^{\infty}$. Since $\overrightarrow{\mathbf{v}}$ is good, we have $w_{0}=\overrightarrow{\mathbf{v}} \#$, which contradicts the consistency of $w_{0}$. Indeed, since $x_{h} R_{\lambda}^{\mathfrak{D}} x_{l}, \eta^{\mathfrak{D}}$ contains $j_{h} \wedge \diamond j_{l}$ as a subformula, maybe preceded by diamonds and conjunctions, and thus $\overrightarrow{\mathbf{v}}^{\#}$ is built from $\left\{\psi_{1}, \ldots, \psi_{m}\right\}$ using $\wedge$ and diamonds and has a subformula $\square_{\lambda} \neg \psi_{i} \wedge \diamond_{\lambda} \psi_{i}$.

Let $d$ be the depth of the spanning tree $\mathfrak{T}$ for $\mathfrak{D}$ used in the construction of $\eta^{\mathfrak{D}}$. We will also use formulas $\gamma_{m}^{\mathfrak{P}}=\square \leq d\left(p_{1} \vee \cdots \vee p_{m}\right) \rightarrow \gamma_{\left\{p_{1}, \ldots, p_{m}\right\}}^{\mathfrak{Q}}$, which are more convenient to work with hereafter. Since $\gamma_{\Psi_{h}}^{\mathfrak{D}}$ is equivalent to a substitution instance of $\gamma_{2^{h}}^{\mathfrak{D}}$, we have

Corollary 3.4. $L^{\mathfrak{D}}$ is axiomatised by $\left\{\gamma_{m}^{\mathfrak{D}} \mid m \in \omega\right\}$.

Intuitively, $\gamma_{m}^{\mathfrak{D}}$ says that if an $d$-neighborhood of a point $w_{0}$ of $\mathfrak{F}$ is coloured in $m$ colours, then we can paint $\tilde{\mathfrak{T}}$ in $m$ colors such that the points of $\tilde{\mathfrak{T}}$ with equal labels have equal colours and there exists a homomorphism from $\tilde{\mathfrak{T}}$ to $\mathfrak{F}$ preserving the colouring and sending $x_{0}$ to $w_{0}$, where $\tilde{\mathfrak{T}}$ is a reduced syntactical tree of $\eta^{\mathfrak{D}}$ defined as follows.

Definition 3.5. Let $\phi$ be a formula built using only $\wedge, \diamond_{\lambda}$ and nominals $j_{k}$ with $0 \leq k \leq n$. A labelled tree with a root $r$ is a tuple $\tilde{\mathfrak{T}}=\left(W,\left(R_{\lambda}: \lambda \in \Lambda\right), r, f\right)$, where $\left(W,\left(R_{\lambda}: \lambda \in \Lambda\right), r\right)$ is a tree with a root $r$ and $f$ (a label function) is a map from $W$ to $\mathcal{P}\left(\left\{x_{0}, \ldots, x_{n}\right\}\right)$. A reduced syntactical tree of a formula $\phi$ is a labelled tree $\tilde{\mathfrak{T}}^{\phi}=\left(W^{\phi},\left(R_{\lambda}^{\phi}: \lambda \in \Lambda\right), r^{\phi}, f^{\phi}\right)$ defined by induction on the length of $\phi$.

Case 1: $\phi=j_{k}$, where $k \in\{0, \ldots, n\}$. Then $W^{\phi}$ contains a single point $y$. The map $f^{\phi}$ takes $y$ to $\left\{x_{k}\right\}$ and the relations $R_{\lambda}^{\phi}$ are empty.

Case 2: $\phi=\chi \wedge \psi$. Then put $W^{\phi}=\left(W^{\chi} \backslash\left\{r^{\chi}\right\}\right) \cup\left(W^{\psi} \backslash\left\{r^{\psi}\right\}\right) \cup\left\{r^{\phi}\right\}$, where $r^{\phi}$ is a new point. The relations $R_{\lambda}$ on $W^{\chi}$ and $W^{\psi}$ remain the same, and $r^{\phi} R_{\lambda} w$ iff $w \in W_{\chi}$ and $r^{\chi} R_{\lambda}^{\chi} w$ or $w \in W^{\psi}$ and $r^{\psi} R_{\lambda}^{\psi} w$. The map $f^{\phi}$ sends $r^{\phi}$ to $f^{\chi}\left(r^{\chi}\right) \cup f^{\psi}\left(r^{\psi}\right)$ and is equal to $f^{\chi}$ or $f^{\psi}$ on all other points.

Case 3: $\phi=\diamond_{\lambda} \psi$. Then $W^{\phi}=W^{\psi} \cup\left\{r^{\phi}\right\}$, where $r^{\phi}$ is a new point. The $R_{\mu}$ for $\mu \neq \lambda$ we leave untouched, and to $R_{\lambda}$ we add an arrow, joining $r^{\phi}$ with $r^{\psi}$. We put $f\left(r^{\phi}\right)=\emptyset$, and do not change $f$ in all other points.

From the definition of $\tilde{\mathfrak{T}}$ and $\eta^{\mathfrak{D}}$ it follows that the label function of $\tilde{\mathfrak{T}}$ maps the points of $\tilde{\mathfrak{T}}$ to singletons, and so it can be understood as a homomorphism from $\tilde{\mathfrak{T}}$ to $\mathfrak{D}$. The labelled tree $\tilde{\mathfrak{T}}$ may be also understood as a sort of unravelling of the initial diagram $\mathfrak{D}$. 

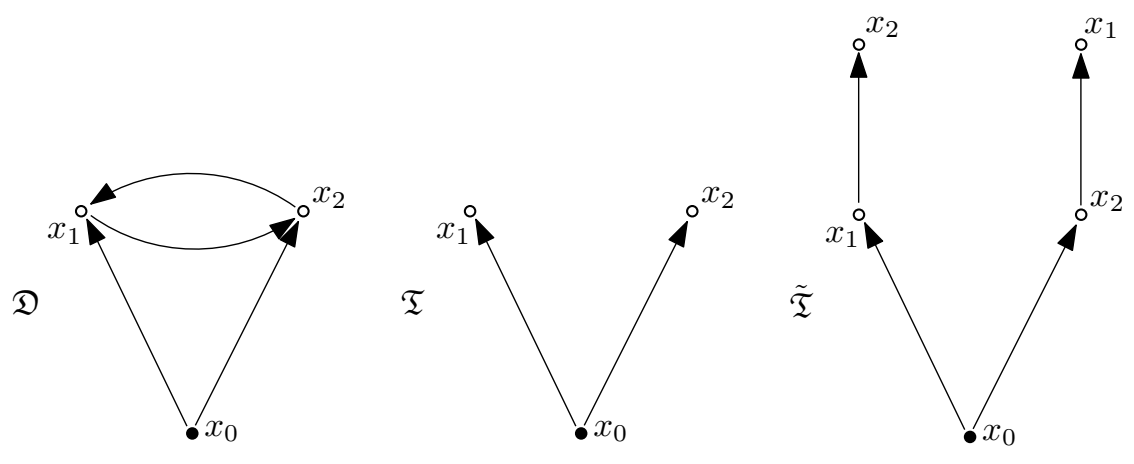

Figure 3: A diagram $\mathfrak{D}$ and its 1-unravelling $\tilde{\mathfrak{T}}$.

Example 3.6. Let $\mathfrak{D}$ be as in the Figure 3 on the left. The spanning tree $\mathfrak{T}$ is in the middle of the Figure 3 , and so $\eta=j_{0} \wedge \diamond\left(j_{2} \wedge \diamond j_{1}\right) \wedge \diamond\left(j_{1} \wedge \diamond j_{2}\right)$, the reduced syntactical tree of which is in the Figure 3 on the right. Thus the logic $L^{\mathfrak{D}}$ is axiomatized by the formulas

$$
\gamma_{m}^{\mathfrak{D}}=\square\left(p_{1} \vee \cdots \vee p_{m}\right) \rightarrow \bigvee_{i, j=1}^{m}\left(\diamond\left(p_{i} \wedge \diamond p_{j}\right) \wedge \diamond\left(p_{j} \wedge \diamond p_{i}\right)\right)
$$

\section{A property of globally minimal diagrams}

Suppose that $\mathfrak{D}$ and $\mathfrak{D}^{\prime}$ are diagrams. A map $f: W^{\mathfrak{D}} \rightarrow W^{\mathfrak{D}^{\prime}}$ is called a monotone map from $\mathfrak{D}$ to $\mathfrak{D}^{\prime}$, if for all $x, y \in W^{\mathfrak{D}}$ and $\lambda \in \Lambda, x R_{\lambda}^{\mathfrak{D}} y$ implies $f(x) R_{\lambda}^{\mathfrak{P}^{\prime}} f(y)$. If also $f\left(x_{0}^{\mathfrak{D}}\right)=x_{0}^{\mathfrak{P}^{\prime}}$, then $f$ is called a homomorphism.

Lemma 4.1. For any globally minimal rooted diagram $\mathfrak{D}$ with an inner cycle, there exist two pointed finite Kripke frames $\mathfrak{F}_{+}^{\mathfrak{D}}=\left(W_{ \pm}^{\mathfrak{P}},\left(\left(R_{+}^{\mathfrak{D}}\right)_{\lambda}: \lambda \in \Lambda\right), w_{0}\right)$ and $\mathfrak{F}_{-}^{\mathfrak{D}}=\left(W_{ \pm}^{\mathfrak{P}},\left(\left(R_{-}^{\mathfrak{D}}\right)_{\lambda}: \lambda \in \Lambda\right), w_{0}\right)$, points $x_{d}, x_{d^{\prime}} \in W^{\mathfrak{D}}$, an index $\lambda_{d} \in \Lambda$, and an injective homomorphism $g: \mathfrak{D} \rightarrow \mathfrak{F}_{+}^{\mathfrak{D}}$ sending $x_{0}$ to $w_{0}$ such that:

$(C-i) \mathfrak{F}_{-}^{\mathfrak{D}}=\mathfrak{F}_{+}^{\mathfrak{D}}-\left(g\left(x_{d}\right), g\left(x_{d}^{\prime}\right), \lambda_{d}\right)$;

(C-ii) $\mathfrak{F}_{-}^{\mathfrak{D}} \not \mathrm{e}^{\mathfrak{D}}\left(w_{0}\right)$;

$\left(C\right.$-iii) $\mathfrak{F}_{+}^{\mathfrak{D}} \models \mathrm{e}^{\mathfrak{D}}\left(w_{0}\right)$;

(C-iv) the points $g\left(x_{d}\right)$ and $g\left(x_{d^{\prime}}\right)$ can be connected in $\mathfrak{F}_{-}^{\mathfrak{P}}$ by an undirected path not passing through $w_{0}$, all points of which belong to the image of $g$;

$(C-v)$ for any homomorphism $h$ from $\mathfrak{D}$ to $\mathfrak{F}_{+}^{\mathfrak{D}}$ we have

$$
\text { the image of } h \text { is }\left\{g\left(x_{0}\right), \ldots, g\left(x_{n}\right)\right\} \text {, }
$$

and for all $0 \leq i, j \leq n$ and $\lambda \in \Lambda$

$$
h\left(x_{i}\right)\left(R_{+}^{\mathfrak{D}}\right)_{\lambda} h\left(x_{j}\right) \text { implies } x_{i} R_{\lambda}^{\mathfrak{D}} x_{j}
$$

(C-vi) if $w \neq w_{0}$, then $\mathfrak{F}_{-}^{\mathfrak{D}} \models \mathrm{e}^{\mathfrak{D}}(w)$ for all $w \in W_{ \pm}^{\mathfrak{D}}$.

$(C$-iv) and $(C-v)$ are technical conditions needed to prove that, for example, the pseudoproducts constructed in Section 5 refute $\mathrm{e}^{\mathfrak{D}}\left(x_{0}\right)$ in their roots. In fact, $(C-v)$ says that any homomorphism $h$ from $\mathfrak{D}$ to $\mathfrak{F}_{+}^{\mathfrak{P}}$ is an isomorphism between $\mathfrak{D}$ and the restriction of $\mathfrak{F}_{+}^{\mathfrak{D}}$ to the image of $h$, and it is easy to see that $(C-v)$ together with $(C-i)$ always imply $\left(C\right.$-ii). Indeed, suppose that $\mathfrak{F}_{-}^{\mathfrak{D}}=\mathrm{e}^{\mathfrak{D}}\left(w_{0}\right)$. Hence, there is a homomorphism $h: \mathfrak{D} \rightarrow \mathfrak{F}_{-}^{\mathfrak{D}}$. But $h$ is also a homomorphism from $\mathfrak{D}$ to $\mathfrak{F}_{+}^{\mathfrak{P}}$. Thus, by $(C-v), h$ is an isomorphism between $\mathfrak{D}$ the restriction of $\mathfrak{F}_{+}^{\mathfrak{D}}$ to $h\left(W^{\mathfrak{D}}\right)$. Therefore there must be points $x_{i}, x_{j} \in \mathfrak{D}$, such that $h\left(x_{i}\right)=g\left(x_{d}\right), h\left(x_{j}\right)=g\left(x_{d^{\prime}}\right)$, and $\left(x_{d}, x_{d^{\prime}}\right) \in R_{\lambda_{d}}^{\mathfrak{D}}$. Now we have a contradiction to the facts that $h$ is a homomorphism to $\mathfrak{F}_{-}^{\mathfrak{D}}$ and $\left(g\left(x_{d}\right), g\left(x_{d^{\prime}}\right)\right) \notin\left(R_{-}^{\mathfrak{P}}\right)_{\lambda_{d}}$. 

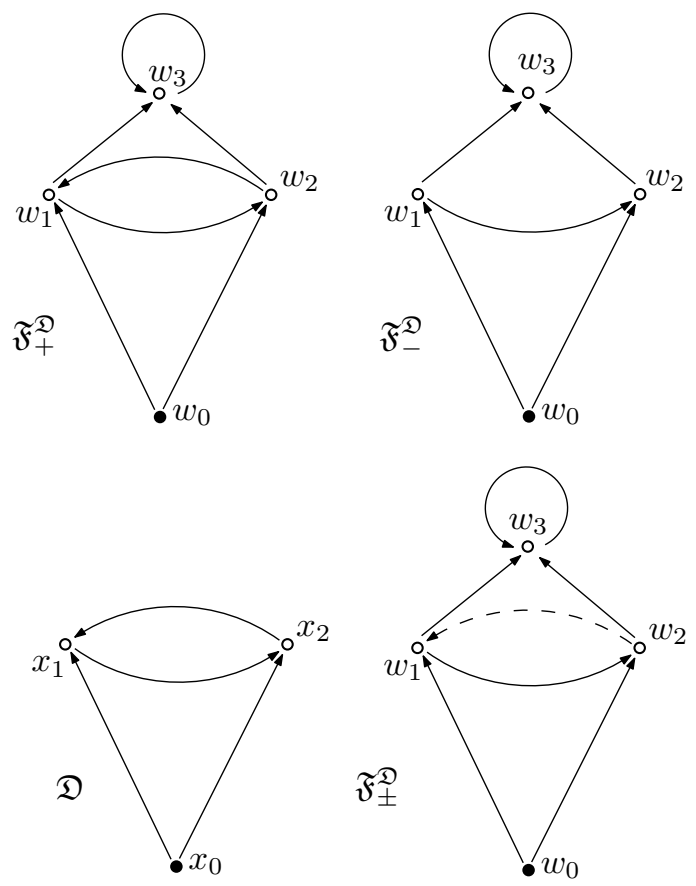

Figure 4:

Example 4.2. Consider the diagram from Figure 4. It is easy to see that the frames $\mathfrak{F}_{+}^{\mathfrak{P}}$ and $\mathfrak{F}_{-}^{\mathfrak{D}}$ from this figure satisfy $(C-i)-\left(C\right.$-vi), where $d=2, d^{\prime}=1$, and $g\left(x_{i}\right)=w_{i}$ for $i=0,1,2$. In particular, there exist two homomorphisms from $\mathfrak{D}$ to $\mathfrak{F}_{+}^{\mathfrak{P}}$ : the first coincides with $g$, the second swaps around $x_{1}$ and $x_{2}$, and both of them satisfy $(C-v)$. Since $\mathfrak{F}_{+}^{\mathfrak{P}}$ and $\mathfrak{F}_{-}^{\mathfrak{D}}$ have a common base set $W_{ \pm}^{\mathfrak{D}}$, one can think of $\mathfrak{F}_{+}^{\mathfrak{P}}$ and $\mathfrak{F}_{-}^{\mathfrak{D}}$ as a tuple $\mathfrak{F}_{ \pm}^{\mathfrak{D}}$, consisting of the frame $\mathfrak{F}_{+}^{\mathfrak{D}}$ and a selected edge given by $d, d^{\prime}$ and $\lambda_{d}$.

In general, to satisfy conditions $(C-i)-(C-v)$, we can always build a spanning tree for $\mathfrak{D}$, and take $\mathfrak{F}^{+}=\mathfrak{D}$ and $\mathfrak{F}^{-}=\mathfrak{F}^{+}-\left(x_{d}, x_{d^{\prime}}, \lambda_{d}\right)$, where $\left(x_{d}, x_{d^{\prime}}, \lambda_{d}\right)$ is one of the edges of the inner cycle not belonging to the spanning tree. The main problem is condition $(C$-vi $)$. In this example we got it at the price of a reflexive point on top of $\mathfrak{D}$. But in some cases this may break the conditions $(C-v)$ and $(C-i i)$, as the next example shows, so a more subtle construction is required.

Example 4.3. Consider the diagram $\mathfrak{D}$ on the left hand side of Figure 5. It is minimal. But if we choose the edge to delete (it could be done in a unique way without affecting connectivity; this edge is dashed in the figure in the middle), add a reflexive point and connect all points except the root to this reflexive point, then the obtained diagram (even after removing the selected arrow) will satisfy $\mathrm{e}_{\mathfrak{D}}\left(x_{0}\right)$ (see the frame in the middle), and that is bad. But we can amend this situation by a more elaborate construction as on the right hand side. Roughly, at first we iteratedly add new points by considering $\mathrm{e}_{\mathfrak{D}}\left(x_{0}\right)$ as a tuple-generating rule, and only after that we close the construction with a reflexive point.

The proof of Lemma 4.1. Given a rooted diagram $\mathfrak{D}=\left(W^{\mathfrak{D}},\left(R_{\lambda}^{\mathfrak{D}}: \lambda \in \Lambda\right), x_{0}\right)$, we define a sequence of tuples $\left(\mathfrak{F}_{i}^{\mathfrak{P}}, A_{i}^{\mathfrak{P}}\right)$ where $\mathfrak{F}_{i}^{\mathfrak{P}}=\left(W_{i}^{\mathfrak{D}}, R_{i, \lambda}^{\mathfrak{D}}, x_{i, 0}^{\mathfrak{D}}\right)$ is a Kripke frame and $A_{i}^{\mathfrak{P}} \subseteq W_{i}^{\mathfrak{P}}$. Intuitively, $A_{i}$ denotes the set of those points $w$ in $\mathfrak{F}_{i}$ which may falsify $(C$-vi) and so have to be "repaired", which results in $\mathfrak{F}_{i+1}$. Formally, we set $\mathfrak{F}_{1}^{\mathfrak{D}}=\mathfrak{D}$ and $A_{1}^{\mathfrak{P}}=W^{\mathfrak{D}} \backslash\left\{x_{0}\right\}, x_{1,0}^{\mathfrak{D}}=x_{0}$. Now given $\left(\mathfrak{F}_{i}^{\mathfrak{D}}, A_{i}^{\mathfrak{D}}\right)$, we define $\left(\mathfrak{F}_{i+1}^{\mathfrak{D}}, A_{i+1}^{\mathfrak{D}}\right)$ as follows. Let

$$
\begin{gathered}
W_{i+1}^{\mathfrak{D}}=\left(\left(W_{i}^{\mathfrak{D}} \backslash A_{i}\right) \times\left\{x_{0}\right\}\right) \cup\left(A_{i} \times W^{\mathfrak{D}}\right), \\
R_{i+1, \lambda}^{\mathfrak{D}}=\left\{\left(\left(a, x_{0}\right),\left(b, x_{0}\right)\right) \mid(a, b) \in R_{i}^{\mathfrak{D}}\right\} \cup\left\{((a, b),(a, c)) \mid a \in A_{i}, b, c \in W^{\mathfrak{D}} \text { and } b R_{\lambda}^{\mathfrak{D}} c\right\}, \\
A_{i+1}^{\mathfrak{D}}=A_{i}^{\mathfrak{D}} \times\left(W^{\mathfrak{D}} \backslash\left\{x_{0}\right\}\right) \text { and } x_{i+1,0}^{\mathfrak{D}}=x_{i, 0}^{\mathfrak{D}} \times\left\{x_{0}\right\} .
\end{gathered}
$$



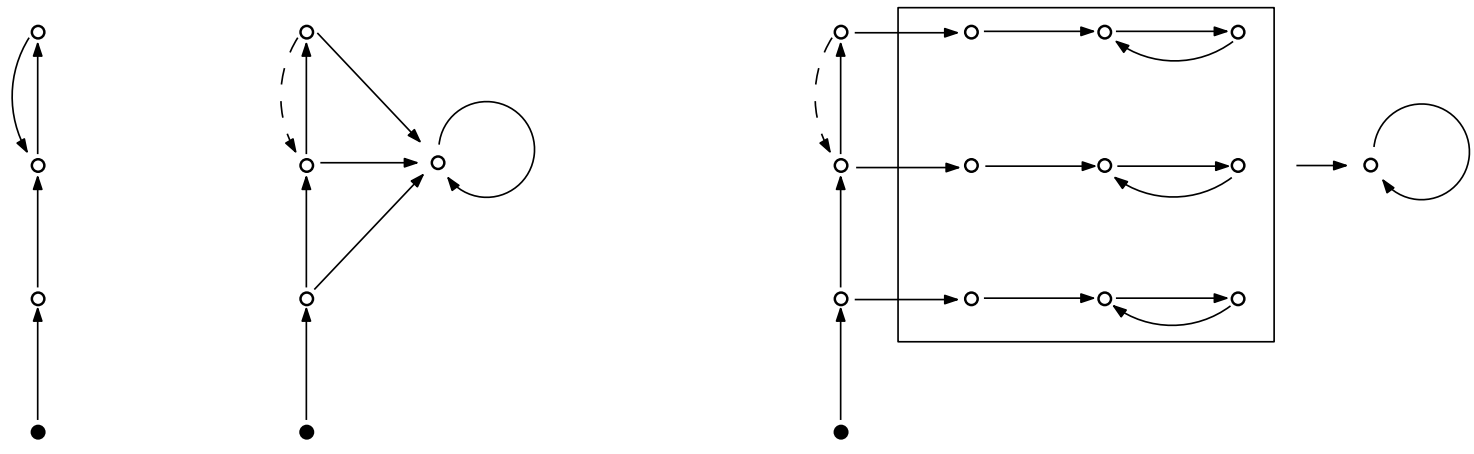

Figure 5:

Let $r$ be the maximal distance from $x_{0}$ to any point $x_{i}$ of $\mathfrak{D}$. To obtain $\mathfrak{F}_{+}^{\mathfrak{D}}$, we take $\mathfrak{F}_{r}^{\mathfrak{D}}$, add a new all- $\lambda$-reflexive point $\circ$, and join all points $y$ of $A_{r}$ to $\circ$ by all $R_{\lambda}$. Now we set $g\left(x_{i}\right)=\left(x_{i}, x_{0}, \ldots, x_{0}\right)$ and $\mathfrak{F}_{-}^{\mathfrak{D}}=\mathfrak{F}_{+}^{\mathfrak{D}}-\left(g\left(x_{d}\right), g\left(x_{d^{\prime}}\right), \lambda_{d}\right)$, where $\left(x_{d}, x_{d^{\prime}}, \lambda_{d}\right)$ is an arbitrary chosen edge of the inner cycle that does not belong to some spanning tree for $\mathfrak{D}$. Denote $\Delta=\left\{g\left(x_{0}\right), \ldots, g\left(x_{n}\right)\right\}=W^{\mathfrak{D}} \times\left\{x_{0}\right\} \times \ldots \times\left\{x_{0}\right\}$. Thus, $g$ is a bijection between $W^{\mathfrak{D}}$ and $\Delta$.

We claim that the conditions $(C-i)-(C$-vi) are satisfied. It is clear that the construction guarantees the validity of conditions $(C-i),\left(C\right.$-iii) and $\left(C\right.$-vi). Condition $\left(C\right.$-iv) is true since $\left(x_{d}, x_{d^{\prime}}, \lambda_{d}\right)$ belongs to the inner cycle.

Let us prove $(C-v)$. Suppose that there exists a homomorphism $h$ from $\mathfrak{D}$ to $\mathfrak{F}_{+}^{\mathfrak{D}}$. It is clear that $h\left(x_{i}\right) \neq \circ$ for all $i$, because $r$ was chosen large enough and so the new reflexive point $\circ$ is too far from $w_{0}$. Let $\Gamma=\Delta \cap\left\{h\left(x_{0}\right), h\left(x_{1}\right), \ldots, h\left(x_{n}\right)\right\}$. Suppose that $\Delta \backslash \Gamma$ is not empty.

For $x_{i} \in W^{\mathfrak{D}}$ let $\operatorname{Rank}\left(x_{i}\right)$ denote the distance from $x_{0}$ to $x_{i}$ in $D$. Let $\operatorname{Del}\left(x_{i}\right)$ be the set of all $x_{j} \in W^{\mathfrak{D}}, x_{j} \neq x_{i}$ such that all paths from $x_{0}$ to $x_{j}$ pass through $x_{i}$. Informally speaking, if we delete $x_{i}$, then $\operatorname{Del}\left(x_{i}\right)$ is the set of all points that cease to be visible from $x_{0}$.

Now let $x_{\alpha}$ be a point of $W^{\mathfrak{D}}$ of maximal rank such that $g\left(x_{\alpha}\right) \in \Delta \backslash \Gamma$. Let $\mathfrak{D}^{\prime}$ be obtained from $\mathfrak{D}$ by deleting $x_{\alpha}$ together with all adjacent edges. The following claims show that $\mathfrak{D}^{\prime}$ is rooted and that the image of $h$ belongs to $\mathfrak{F}_{r}^{\mathfrak{D}^{\prime}}$ considered as a part of $\mathfrak{F}_{r}^{\mathfrak{D}}$.

Claim 1. $\operatorname{Del}\left(x_{\alpha}\right)=\emptyset$. Otherwise, take a point $x_{\alpha^{\prime}}$ of $\operatorname{Del}\left(x_{\alpha}\right)$. From the definition of $\operatorname{Del}\left(x_{\alpha}\right)$ and $\mathfrak{F}_{r}^{\mathfrak{D}}$ it follows that all paths in $\mathfrak{F}_{+}^{\mathfrak{D}}$ leading from $g\left(x_{0}\right)$ to $g\left(x_{\alpha^{\prime}}\right)$ pass through $g\left(x_{\alpha}\right)$. Let us prove that $g\left(x_{\alpha^{\prime}}\right) \notin \Gamma$. Suppose that $g\left(x_{\alpha^{\prime}}\right) \in \Gamma$, that is $g\left(x_{\alpha^{\prime}}\right)=h\left(x_{\alpha^{\prime \prime}}\right)$ for some $\alpha^{\prime \prime}$. Since $\mathfrak{D}$ is rooted, there exists a path in $\mathfrak{D}$ from $x_{0}$ to $x_{\alpha^{\prime \prime}}$. The image of this path under the map $h$ is a path connecting $g\left(x_{0}\right)$ to $g\left(x_{\alpha^{\prime}}\right)$ in $\mathfrak{F}_{+}^{\mathfrak{D}}$. But this path must pass through $g\left(x_{\alpha}\right)$. That contradicts $g\left(x_{\alpha}\right) \notin \Gamma$. Hence, $g\left(x_{\alpha^{\prime}}\right) \notin \Gamma$. $\operatorname{But} \operatorname{Rank}\left(x_{\alpha^{\prime}}\right)>\operatorname{Rank}\left(x_{\alpha}\right)$. This contradicts the maximality of the rank of $x_{\alpha}$ in $\Delta \backslash \Gamma$.

Claim 2. If $h\left(x_{\alpha}\right)=\left(x_{i_{1}}, \ldots, x_{i_{n}}\right)$, then $x_{i_{k}} \neq x_{\alpha}$ for all $1 \leq k \leq n$. First, consider the case when $h\left(x_{\alpha}\right) \in \Delta$. Then there is nothing to prove, because in this case $i_{k}=0$ for $2 \leq k \leq n$, and $i_{1} \neq \alpha$ since $g\left(x_{\alpha}\right) \notin \Gamma$. So, suppose that $h\left(x_{\alpha}\right) \notin \Delta$. This means that

$$
\left|\left\{k \mid x_{i_{k}} \neq x_{0}\right\}\right| \geq 2
$$

Let us prove that for all $1 \leq k \leq n$ we have $\operatorname{Rank}\left(x_{i_{k}}\right)<\operatorname{Rank}\left(x_{\alpha}\right)$. To this end, note that the distance in $\mathfrak{F}_{+}^{\mathfrak{D}}$ satisfies

$$
\operatorname{Dist}_{\mathfrak{F}_{+}^{\mathfrak{P}}}\left(\left(x_{0}, \ldots, x_{0}\right),\left(x_{i_{1}}, \ldots, x_{i_{n}}\right)\right)=\operatorname{Dist}_{\mathfrak{D}}\left(x_{0}, x_{i_{1}}\right)+\ldots+\operatorname{Dist}_{\mathfrak{D}}\left(x_{0}, x_{i_{n}}\right)
$$

Now, suppose that for some $k$ we have $\operatorname{Rank}\left(x_{i_{k}}\right) \geq \operatorname{Rank}\left(x_{\alpha}\right)$. Then taking into account (V) we obtain that

$$
\operatorname{Dist}_{\mathfrak{F}_{+}^{\mathfrak{P}}}\left(w_{0}, h\left(x_{\alpha}\right)\right)=\operatorname{Dist}_{\mathfrak{F}_{+}^{\mathfrak{P}}}\left(\left(x_{0}, \ldots, x_{0}\right),\left(x_{i_{1}}, \ldots, x_{i_{n}}\right)\right)>\operatorname{Rank}\left(x_{\alpha}\right),
$$

a contradiction. Hence, $\operatorname{Rank}\left(x_{i_{k}}\right)<\operatorname{Rank}\left(x_{\alpha}\right)$ for all $1 \leq k \leq n$, and, in particular, $x_{i_{k}} \neq x_{\alpha}$.

Claim 3. For any $\beta \in\{1, \ldots, n\}$, if $h\left(x_{\beta}\right)=\left(x_{i_{1}}, \ldots, x_{i_{n}}\right)$, then $x_{i_{k}} \neq x_{\alpha}$ for all $1 \leq k \leq n$. Indeed, if $\operatorname{Rank}\left(x_{\beta}\right) \leq \operatorname{Rank}\left(x_{\alpha}\right)$, then a similar argument works. Now, $\operatorname{suppose}$ that $\operatorname{Rank}\left(x_{\beta}\right)>\operatorname{Rank}\left(x_{\alpha}\right)$. Let $B \subseteq W^{\mathfrak{D}}$ be the set of all points of rank greater than $\operatorname{Rank}\left(x_{\alpha}\right)$. But as $x_{\alpha}$ is supposed to be an element 
of $\Delta \backslash \Gamma$ of maximal rank, from the definition of $B$ it follows that $h(B) \subseteq \Delta$, and, in particular, for all $k>2 x_{i_{k}}=x_{0} \neq x_{\alpha}$.

Now, let the diagram $\mathfrak{D}^{\prime}$ be obtained from $\mathfrak{D}$ by deleting $x_{\alpha}$ together with all adjacent edges. Then $\vdash_{F O L} \forall x_{0} \mathrm{e}^{\mathfrak{D}^{\prime}}\left(x_{0}\right) \rightarrow \forall x_{0} \mathrm{e}^{\mathfrak{F}_{r}^{\mathfrak{P}^{\prime}}}\left(x_{0}\right)$ and $\vdash_{F O L} \forall x_{0} \mathrm{e}^{\mathfrak{F}_{r}^{\mathfrak{P}^{\prime}}}\left(x_{0}\right) \rightarrow \forall x_{0} \mathrm{e}^{\mathfrak{D}}\left(x_{0}\right)$. Thus $\vdash_{F O L} \forall x_{0} \mathrm{e}^{\mathfrak{D}^{\prime}}\left(x_{0}\right) \rightarrow$ $\forall x_{0} \mathrm{e}^{\mathfrak{D}}\left(x_{0}\right)$, and this contradicts the global minimality of $\mathfrak{D}$.

We have just proved (III) of $(C-v)$. To prove (IV), take a homomorphism $h$ from $\mathfrak{D}$ to $\mathfrak{F}_{\mathfrak{D}}^{+}$. Via identification the $\mathfrak{D}$ with a copy of itself sitting inside $\mathfrak{F}_{\mathfrak{D}}^{+}$given by the image of $g$, we see that the map $h$ acts on the set $\operatorname{Arr}(\mathfrak{D})=\left\{\left(x_{i}, x_{j}, \lambda\right) \mid x_{i}, x_{j} \in W^{\mathfrak{D}}, \lambda \in \Lambda,\left(x_{i}, x_{j}\right) \in R_{\lambda}^{\mathfrak{D}}\right\}$, sending $\left(x_{i}, x_{j}, \lambda\right)$ to $\left(h\left(x_{i}\right), h\left(x_{j}\right), \lambda\right)$ which is also in $\operatorname{Arr}(\mathfrak{D})$. From (III) it follows that $h$ is injective on $\operatorname{Arr}(\mathfrak{D})$. Therefore, since $\operatorname{Arr}(\mathfrak{D})$ is finite, $h$ is surjective on $\operatorname{Arr}(\mathfrak{D})$, and so satisfies (IV) of $(C-v)$.

Another proof of (IV) of $(C-v)$ : take a homomorphism $h$ from $\mathfrak{D}$ to $\mathfrak{F}_{\mathfrak{D}}^{+}$and assume that $h\left(x_{i}\right)\left(R_{+}^{\mathfrak{D}}\right)_{\lambda} h\left(x_{j}\right)$ holds while $x_{i} R_{\lambda}^{\mathfrak{P}} x_{j}$ does not. It follows that the edge $g^{-1}\left(h\left(x_{i}\right)\right) R_{\lambda}^{\mathfrak{D}} g^{-1}\left(h\left(x_{i}\right)\right)$ (which is well defined because of (III)) is superfluous in $\mathfrak{D}$.

Condition $(C-i i)$ is a consequence of $(C-v)$ and $(C-i)$.

\section{Pseudoproducts with graphs}

By a graph we understand a tuple $G=(V, E)$, where $E$ is a symmetric binary relation on $V$. To emphasise symmetricity of $E$, instead of $\left(v_{1}, v_{2}\right) \in E$ we sometimes write $\left\{v_{1}, v_{2}\right\} \in E$. For a ordinal $\alpha \leq \omega$ an $\alpha$-colouring of a graph $G$ is a map $\tau: V \rightarrow \alpha$, such that every two adjacent vertices are mapped to different elements of $\alpha$. The elements of $\alpha$ in this context are called colours. In general, below we do not suppose $E$ to be irreflexive, thus $G$ may contain edges of the form $(v, v)$, which are called loops. However, it is clear that any graph that contains loops does not have colourings at all, and so in Lemma 5.1, (C2) we implicitly assume that $G$ does not have loops.

We fix a minimal diagram $\mathfrak{D}$ with an inner cycle. Let $\mathfrak{F}_{+}^{\mathfrak{P}}=\left(W_{ \pm}^{\mathfrak{P}},\left(\left(R_{+}^{\mathfrak{P}}\right)_{\lambda}: \lambda \in \Lambda\right), w_{0}\right)$ and $\mathfrak{F}_{-}^{\mathfrak{D}}=$ $\left(W_{ \pm}^{\mathfrak{P}},\left(\left(R_{-}^{\mathfrak{D}}\right)_{\lambda}: \lambda \in \Lambda\right), w_{0}\right)$ together with $d, d^{\prime}, \lambda_{d}$ and $g$ satisfy conditions $(C-i)-(C$-vi) of Lemma 4.1 . Let $G=(V, E)$ be an arbitrary graph. By $\mathfrak{F}_{ \pm}^{\mathfrak{P}} \times G$ denote $^{3}$ the Kripke frame $\left(W^{\mathfrak{D}, G},\left(R_{\lambda}^{\mathfrak{D}, G}: \lambda \in \Lambda\right)\right)$ where $W^{\mathfrak{D}, G}=\left\{w_{0}\right\} \cup\left(W_{ \pm}^{\mathfrak{D}} \backslash\left\{w_{0}\right\}\right) \times V$ and

$$
\begin{gathered}
R_{\lambda}^{\mathfrak{D}, G}=\left\{\left(w_{0}, w_{0}\right) \mid \mathfrak{F}_{-}^{\mathfrak{D}} \models w_{0} R_{\lambda} w_{0} ;\right\} \cup \\
\left\{\left(w_{0},(y, v)\right) \mid \mathfrak{F}_{-}^{\mathfrak{D}} \models w_{0} R_{\lambda} y ; y \in W_{ \pm}^{\mathfrak{D}} \backslash\left\{w_{0}\right\} ; v \in V\right\} \cup \\
\left\{\left((y, v), w_{0}\right) \mid \mathfrak{F}_{-}^{\mathfrak{D}} \models y R_{\lambda} w_{0} ; y \in W_{ \pm}^{\mathfrak{D}} \backslash\left\{w_{0}\right\} ; v \in V\right\} \cup \\
\left\{((x, v),(y, v)) \mid \mathfrak{F}_{-}^{\mathfrak{D}} \models x R_{\lambda} y ; x, y \in W_{ \pm}^{\mathfrak{D}} \backslash\left\{w_{0}\right\} ; v \in V ;\right\} \cup \\
\left\{\left(\left(g\left(x_{d}\right), v_{1}\right),\left(g\left(x_{d^{\prime}}\right), v_{2}\right)\right) \mid v_{1} \in V ; v_{2} \in V ;\left\{v_{1}, v_{2}\right\} \in E, \lambda=\lambda_{d}\right\} .
\end{gathered}
$$

An example of this construction for $\mathfrak{D}$ and $\mathfrak{F}_{ \pm}^{\mathfrak{P}}$ from Example 4.2 is given in Figure 6. This construction has a simpler description in terms of projection functions. If $p r$ denotes the projection from $\mathfrak{F}_{ \pm}^{\mathfrak{P}} \times G$ to $\mathfrak{F}_{+}^{\mathfrak{P}}$, given by formulas $\operatorname{pr}((x, v))=x, \operatorname{pr}\left(w_{0}\right)=w_{0}$, and $h$ denotes the projection from $\mathfrak{F}_{ \pm}^{\mathfrak{P}} \times G$ to $V^{\prime}$, where $V^{\prime}=V \cup\{\perp\}$, given by formulas $h((x, v))=v, h\left(w_{0}\right)=\perp$, then the $R_{\lambda}^{\mathfrak{D}, G}$ satisfy the following condition for all $\eta, \chi \in W^{\mathfrak{D}, G}: \mathfrak{F}_{ \pm}^{\mathfrak{D}} \times G \models \eta R_{\lambda} \chi$ iff

$$
\begin{gathered}
\mathfrak{F}_{-}^{\mathfrak{D}} \models \operatorname{pr}(\eta) R_{\lambda} \operatorname{pr}(\chi) \text { and }(h(\eta)=h(\chi) \text { or } \perp \in\{h(\eta), h(\chi)\}) \\
\mathfrak{F}_{-}^{\mathfrak{D}} \not \models \operatorname{pr}(\eta) R_{\lambda} \operatorname{pr}(\chi), \mathfrak{F}_{+}^{\mathfrak{P}} \models \operatorname{pr}(\eta) R_{\lambda} \operatorname{pr}(\chi) \text { and } G=h(\eta) E h(\chi) .
\end{gathered}
$$

Recall that $L^{\mathfrak{D}}$ is axiomatized by formulas $\gamma_{n}^{\mathfrak{D}}$ of Section 3 saying "if an d-neighborhood of a point $w_{0}$ of $\mathfrak{F}$ is painted in $m$ colours, then we can paint the tree $\tilde{\mathfrak{T}}=\left(W^{\tilde{\mathfrak{T}}},\left(R_{\lambda}^{\tilde{\mathfrak{T}}}: \lambda \in \Lambda\right), x_{0}\right)$ (defined in Section 3$)$ in $m$ colors such that the points of $\tilde{\mathfrak{T}}$ with equal labels have equal colours and there exists a homomorphism from $\tilde{\mathfrak{T}}$ to $\mathfrak{F}$ sending $x_{0}$ to $w_{0}$ and preserving the colouring". We understand $f^{\tilde{\mathfrak{T}}}$ as a homomorphism from $\tilde{\mathfrak{T}}$ to $\mathfrak{D}$.

\footnotetext{
${ }^{3}$ We chose this notation for pseudoproducts because they somehow combine the features of $\mathfrak{F}_{+}^{\mathfrak{D}}$ and $\mathfrak{F}_{-}^{\mathfrak{D}}$. You may think of $\mathfrak{F}_{ \pm}^{\mathfrak{D}}$ as a shorthand for a tuple $\left(\mathfrak{F}_{+}^{\mathfrak{D}}, \mathfrak{F}_{-}^{\mathfrak{D}}\right)$ with intuition from Example 4.2.
} 


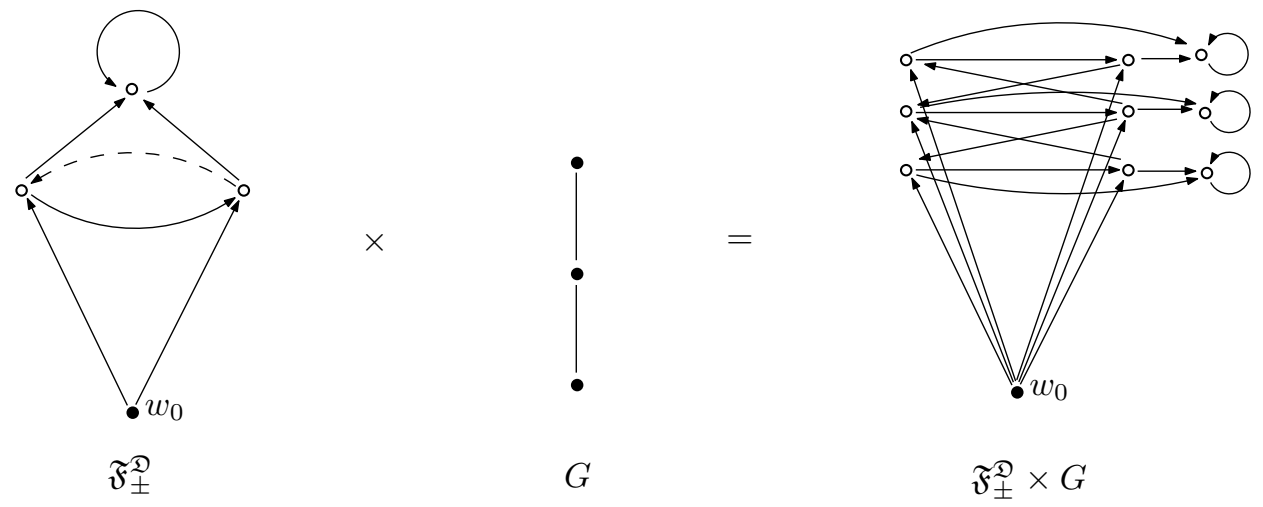

Figure 6: A pseudoproduct.

The next lemma shows the connection between the chromatic number of $G$ and the least $k$ for which $\gamma_{k}^{\mathfrak{P}}$ can be refuted in $\mathfrak{F}_{ \pm}^{\mathfrak{P}} \times G$. Simply put, it says that if one of these numbers is big, then the other is big as well.

Lemma 5.1. Suppose that $\left|W_{ \pm}^{\mathfrak{P}}\right|=b$ and that $G=(V, E)$. Then

(C1) If $G$ cannot be painted in $2^{b k}$ colours, then for all $k$-generated valuations $\theta$ and for all $m$ we have $\mathfrak{F}_{ \pm}^{\mathfrak{D}} \times G, \theta \models \gamma_{m}^{\mathfrak{D}}$. In particular, $\mathfrak{F}_{ \pm}^{\mathfrak{P}} \times G \models \gamma_{k}^{\mathfrak{D}}$.

(C2) If $G$ can be painted in $N$ colours, then $\mathfrak{F}_{ \pm}^{\mathfrak{P}} \times G \not \models \gamma_{N(b-1)+1}^{\mathfrak{D}}$.

Proof. (C1) Condition (C-vi) of Lemma 4.1 and the soundness part of Theorem 3.3 guarantee that $\mathfrak{F}_{ \pm}^{\mathfrak{P}} \times G, x \models \gamma_{m}^{\mathfrak{P}}$ for all $x \in W^{\mathfrak{D}, G}$ different from $w_{0}$. We show that $\mathfrak{F}_{ \pm}^{\mathfrak{P}} \times G, \theta, w_{0} \models \gamma_{m}^{\mathfrak{D}}$ as well, if $\theta$ is $k$-generated. Since a formula $\gamma_{m}^{\mathcal{D}}$ is invariant under transpositions of variables that swap $p_{i}$ and $p_{j}$, without any loss of generality we may assume that $\theta\left(p_{i}\right)=\emptyset$ for $i>k$. Define the map $\tau: V \rightarrow(\mathcal{P}(\{1, \ldots, k\}))^{(b-1)}$ by putting $\tau(v)$ for $v \in V$ to be the map from $W_{ \pm}^{\mathfrak{P}} \backslash\left\{w_{0}\right\}$ to $\mathcal{P}(\{1, \ldots, k\})$ defined by

$$
i \in \tau(v)(y) \text { iff }(y, v) \in \theta\left(p_{i}\right) \quad \text { for } 1 \leq i \leq k .
$$

Since $G$ cannot be painted in $2^{b k}$ colours, there exist $v_{1}, v_{2} \in V$ such that $\left\{v_{1}, v_{2}\right\} \in E$ and $\tau\left(v_{1}\right)=\tau\left(v_{2}\right)$. Consider the Kripke frame $\left(\mathfrak{F}_{ \pm}^{\mathfrak{P}} \times G\right)^{\dagger}=\left(\left(W^{\mathfrak{D}, G}\right)^{\dagger},\left(R_{\lambda}^{\mathfrak{D}, G}\right)^{\dagger}, w_{0}\right)$, where $\left(W^{\mathfrak{D}, G}\right)^{\dagger}=W^{\mathfrak{D}, G} \cup W_{ \pm}^{\mathfrak{P}}$ (recall that $\left.W_{ \pm}^{\mathfrak{P}} \cap W^{\mathfrak{D}, G}=\left\{w_{0}\right\}\right)$ and $\left(R_{\lambda}^{\mathfrak{D}, G}\right)^{\dagger}=R_{\lambda}^{\mathfrak{D}, G} \cup\left(R_{+}^{\mathfrak{D}}\right)_{\lambda}$. Then we set

$$
\theta^{\dagger}\left(p_{i}\right)=\theta\left(p_{i}\right) \cup\left\{y \in W_{ \pm}^{\mathfrak{D}} \mid\left(y, v_{1}\right) \in \theta\left(p_{i}\right)\right\}
$$

for all $1 \leq i \leq k$. We claim that $\left(\left(\mathfrak{F}_{ \pm}^{\mathfrak{P}} \times G\right)^{\dagger}, \theta^{\dagger}\right), w_{0}$ and $\left(\mathfrak{F}_{ \pm}^{\mathfrak{P}} \times G, \theta\right), w_{0}$ are bisimilar. Indeed, the relation $Z=\left\{(z, z) \mid z \in W^{\mathfrak{D}, G}\right\} \cup\left\{\left(y,\left(y, v_{1}\right)\right) \mid y \in W_{ \pm}^{\mathfrak{P}} \backslash\left\{w_{0}\right\}\right\} \cup\left\{\left(y,\left(y, v_{2}\right)\right) \mid y \in W_{ \pm}^{\mathfrak{D}} \backslash\left\{w_{0}\right\}\right\}$ constitutes a bisimulation. Since $\left(\mathfrak{F}_{ \pm}^{\mathfrak{P}} \times G\right)^{\dagger}, \theta^{\dagger}, w_{0} \models \gamma_{m}^{\mathfrak{D}}$ (Theorem 3.3, Soundness), we conclude that also $\mathfrak{F}_{ \pm}^{\mathfrak{P}} \times G, \theta, w_{0} \models \gamma_{m}^{\mathfrak{P}}$.

(C2) Let $\tau: V \rightarrow\{1, \ldots, N\}$ be a colouring of $G$. Suppose that the variables of $\gamma_{N(b-1)+1}^{\mathfrak{D}}$ are indexed as $p_{0}$ and $p_{i}^{c}$ where $1 \leq i \leq b-1,1 \leq c \leq N$. Consider the following valuation $\theta$ on $\mathfrak{F}_{ \pm}^{\mathfrak{P}} \times G$ :

$$
\theta(p)= \begin{cases}\left\{x_{0}\right\}, & \text { if } p=p_{0}, \\ \left\{\left(x_{i}, v\right) \mid \tau(v)=c\right\}, & \text { if } p=p_{i}^{c} .\end{cases}
$$

The definition of $\theta$ gives rise to the map $\theta_{*}: \mathfrak{F}_{ \pm}^{\mathfrak{P}} \times G \rightarrow\{0,1, \ldots, N(b-1)\}$ defined by equations $\theta_{*}\left(w_{0}\right)=0 ; \theta_{*}((y, v))=$ the number of $p_{i}^{\tau(v)}$ among $\{1, \ldots, N(b-1)\}$.

Recall that there is a natural projection $p r: \mathfrak{F}_{ \pm}^{\mathfrak{P}} \times G \rightarrow \mathfrak{F}_{+}^{\mathfrak{P}}$, defined by

$$
\begin{gathered}
\operatorname{pr}\left(w_{0}\right)=w_{0} ; \\
\operatorname{pr}\left(x_{i}, v\right)=x_{i} \text { for all } v \in V .
\end{gathered}
$$




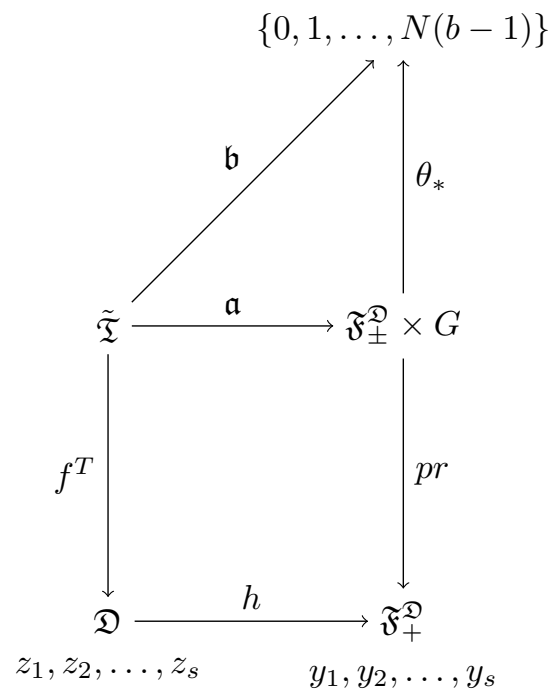

Figure 7:

Besides $p r$, there is a projection $f^{\tilde{\mathfrak{T}}}: \tilde{\mathfrak{T}} \rightarrow \mathfrak{D}$. We say that a map $\mathfrak{b}: W^{\tilde{\mathfrak{T}}} \rightarrow\{0,1, \ldots, N(b-1)\}$ respects $f^{\tilde{\mathfrak{T}}}$ if for all $x, y \in W^{\tilde{\mathfrak{T}}}, f^{\tilde{\mathfrak{L}}}(x)=f^{\tilde{\mathfrak{T}}}(y)$ implies $\mathfrak{b}(x)=\mathfrak{b}(y)$.

To prove that $\mathfrak{F}_{ \pm}^{\mathfrak{D}} \times G, \theta \not \models \gamma_{N(b-1)+1}^{\mathfrak{D}}$ it is sufficient to prove that there is no homomorphism $\mathfrak{a}: \tilde{\mathfrak{T}} \rightarrow$ $\mathfrak{F}_{ \pm}^{\mathfrak{P}} \times G$, such that $\theta_{*}(\mathfrak{a}(t)): \tilde{\mathfrak{T}} \rightarrow\{0,1, \ldots, N(b-1)\}$ respects $f^{\tilde{\mathfrak{T}}}$.

For the sake of contradiction, assume that such $\mathfrak{a}$ exists. Consider the following map $h: W^{\mathfrak{D}} \rightarrow W_{ \pm}^{\mathfrak{D}}$. To define $h(x)$ for $x \in W^{\mathfrak{D}}$, we take any $t \in\left(f^{\tilde{\mathfrak{T}}}\right)^{-1}(x)$ and set $h(x)=\operatorname{pr}(\mathfrak{a}(t)$ ) (see Figure 7). It is clear that $h$ is well defined, i.e., it does not depend on the choice of $t$, since if $t_{1}, t_{2} \in\left(f^{\tilde{\mathfrak{T}}}\right)^{-1}(x)$, then $f^{\tilde{\mathfrak{T}}}\left(t_{1}\right)=f^{\tilde{\mathfrak{T}}}\left(t_{2}\right)$, and this means that $\operatorname{pr}\left(\mathfrak{a}\left(t_{1}\right)\right)=\operatorname{pr}\left(\mathfrak{a}\left(t_{2}\right)\right)$ due to the definition of $\theta$. Clearly, $h$ is a homomorphism from $\mathfrak{D}$ to $\mathfrak{F}_{+}^{\mathfrak{D}}$, and it makes the diagram in Figure 7 commutative. Now we apply $(C-v)$ of Lemma 4.1 and conclude that the image of $h$ is $\left\{g\left(x_{0}\right), g\left(x_{1}\right), \ldots, g\left(x_{n}\right)\right\}$.

Then we apply $(C-i v)$. Let $y_{1} R_{\lambda_{1}} y_{2} \ldots R_{\lambda_{n-1}} y_{s}$, where $y_{i} \in W_{ \pm}^{\mathfrak{P}}$ for $1 \leq i \leq s$ and $\lambda_{i} \in \Lambda^{ \pm}$for $1 \leq i<s$, be the path connecting $x_{d}$ with $x_{d^{\prime}}$ in $\mathfrak{F}_{-}^{\mathfrak{D}}$ (in particular, $y_{1}=x_{d}$ and $y_{s}=x_{d^{\prime}}$ ). Let $z_{1}, \ldots, z_{s} \in W^{\mathfrak{D}}$ be the points such that $h\left(z_{i}\right)=y_{i}$ for $1 \leq i \leq s$. By $(C-v),(\mathrm{IV}), \mathfrak{D} \models z_{i} R_{\lambda_{i}} z_{i+1}$ for $1 \leq i<s$. The map $f^{\tilde{\mathfrak{T}}}$ satisfies the following condition: if $\mathfrak{D}=w_{1} R_{\lambda} w_{2}$ for some $w_{1}, w_{2} \in W^{\mathfrak{D}}$, then there exist points $w_{1}^{\prime}, w_{2}^{\prime} \in W^{\tilde{\mathfrak{T}}}$ such that $\tilde{\mathfrak{T}} \models w_{1}^{\prime} R_{\lambda} w_{2}^{\prime}$ and $w_{i}^{\prime} \in\left(f^{\tilde{\mathfrak{T}}}\right)^{-1}\left(w_{i}\right)$ for $i \in\{1,2\}$. We apply this statement $s-1$ times for $z_{i}, R_{\lambda_{i}}$ and $z_{i+1}$, and conclude that there exist points $t_{i} \in\left(f^{\tilde{\mathfrak{T}}}\right)^{-1}\left(z_{i}\right)$ and $t_{j}^{\prime} \in\left(f^{\tilde{\mathfrak{T}}}\right)^{-1}\left(z_{j}\right)$ for $1 \leq i<s$ and $1<j \leq s$ such that $\tilde{\mathfrak{T}} \models t_{i} R_{\lambda_{i}} t_{i+1}^{\prime}$ for $1 \leq i<s$. Note that by the definition of $t_{i}$ and $t_{i}^{\prime}$ we have $\operatorname{pr}\left(\mathfrak{a}\left(t_{i}\right)\right)=\operatorname{pr}\left(\mathfrak{a}\left(t_{i}^{\prime}\right)\right)=y_{i}$.

Thus, let $v_{i} \in V$ and $v_{j}^{\prime} \in V$ for $1 \leq i<s$ and $1<j \leq s$ be such that $\mathfrak{a}\left(t_{i}\right)=\left(y_{i}, v_{i}\right)$ and $\mathfrak{a}\left(t_{j}^{\prime}\right)=\left(y_{i}, v_{j}^{\prime}\right)$. Let us show that

(a) if $1 \leq i<s$ then $\tau\left(v_{i}\right)=\tau\left(v_{i+1}^{\prime}\right)$ and

(b) if $1<i<s$ then $\tau\left(v_{i}\right)=\tau\left(v_{i}^{\prime}\right)$.

(a): Since $\tilde{\mathfrak{T}} \models t_{i} R_{\lambda_{i}} t_{i+1}$, due to the definition of $R^{\mathfrak{D}, G}$ and the facts that $\left(f^{\tilde{\mathfrak{T}}}\left(t_{i}\right), f^{\tilde{\mathfrak{T}}}\left(t_{i+1}\right)\right) \in\left(R_{-}^{\mathfrak{D}}\right)_{\lambda_{i}}$ and $\left(g\left(x_{d}\right), g\left(x_{d^{\prime}}\right)\right) \notin\left(R_{-}^{\mathfrak{D}}\right)_{\lambda_{d}}$, it follows that $v_{i}=v_{i+1}^{\prime}$, and so $\tau\left(v_{i}\right)=\tau\left(v_{i+1}^{\prime}\right)$.

(b): From $t_{i}, t_{i}^{\prime} \in\left(f^{\tilde{\mathfrak{T}}}\right)^{-1}\left(z_{i}\right)$ and the fact that $\theta(\mathfrak{a}(t))$ respects $f^{\tilde{\mathfrak{T}}}$, it follows that $\theta_{*}\left(\mathfrak{a}\left(t_{i}\right)\right)=\theta_{*}\left(\mathfrak{a}\left(t_{i}^{\prime}\right)\right)$. Therefore $\theta_{*}\left(\left(y_{i}, v_{i}\right)\right)=\theta_{*}\left(\left(y_{i}, v_{i}^{\prime}\right)\right)$, and so $\tau\left(v_{i}\right)=\tau\left(v_{i}^{\prime}\right)$.

Together, (a) and (b) give us that $\tau\left(v_{1}\right)=\tau\left(v_{s}^{\prime}\right)$. Since $F_{+}^{\mathfrak{D}} \models g\left(x_{d}\right) R_{\lambda_{d}} g\left(x_{d}^{\prime}\right),(C-v)$ implies that $\mathfrak{D}=z_{1} R_{\lambda_{d}} z_{s}$, and so there exist $t_{1}^{\prime} \in\left(f^{\tilde{\mathfrak{T}}}\right)^{-1}\left(z_{1}\right)$ and $t_{s} \in\left(f^{\tilde{\mathfrak{T}}}\right)^{-1}\left(z_{s}\right)$ such that $\tilde{\mathfrak{T}} \models t_{1}^{\prime} R_{\lambda_{d}} t_{s}$. Let $v_{1}^{\prime}$ and $v_{s}$ be such that $\mathfrak{a}\left(t_{1}^{\prime}\right)=\left(y_{1}, v_{1}^{\prime}\right)$ and $\mathfrak{a}\left(t_{s}\right)=\left(y_{s}, v_{s}\right)$. Arguing like in (b), one can show that $\tau\left(v_{s}\right)=\tau\left(v_{s}^{\prime}\right)$ and that $\tau\left(v_{1}\right)=\tau\left(v_{1}^{\prime}\right)$. But $\tilde{\mathfrak{T}}=t_{1}^{\prime} R_{\lambda_{d}} t_{s}$ together with the facts that $\mathfrak{a}$ is a homomorphism and that $\tau$ is a colouring of $G$ imply that $\tau\left(v_{1}\right) \neq \tau\left(v_{s}^{\prime}\right)$ (recall that $y_{1}=x_{d}$ and $y_{s}=x_{d}^{\prime}$ ), a contradiction. 


\section{Pseudoproducts with complete graphs}

Fix a diagram $\mathfrak{D}$. For an ordinal $\alpha$ let $K_{\alpha}$ denote the complete graph with $\alpha$ vertices.

Lemma 6.1. For any $\alpha \mathfrak{F}_{ \pm}^{\mathfrak{P}} \times K_{\alpha} \not \neq \mathrm{e}^{\mathfrak{D}}\left(w_{0}\right)$.

Proof. For finite $\alpha$ this is a consequence of Lemma 5.1, (C2) and the fact that $\mathfrak{F}=\mathrm{e}^{\mathfrak{D}}(x)$ implies $\mathfrak{F}=\gamma_{i}^{\mathfrak{D}}$ for all $i \in \omega$ and every Kripke frame $\mathfrak{F}$ (Theorem 3.3, Soundness). Then notice that if $\mathfrak{F}_{ \pm}^{\mathfrak{P}} \times K_{\alpha} \models \mathrm{e}^{\mathfrak{D}}\left(w_{0}\right)$ for infinite $\alpha$, then $\mathfrak{F}_{ \pm}^{\mathfrak{P}} \times K_{\alpha^{\prime}} \models \mathrm{e}^{\mathfrak{D}}\left(w_{0}\right)$ for some finite $\alpha^{\prime}$, because of the form of $\mathrm{e}^{\mathfrak{D}}\left(x_{0}\right)$.

For a point $z \in W^{\mathfrak{D}, K_{\alpha}}$ let $\pi_{z}$ denote the pricipal ultrafilter corresponding to the point $z$.

Lemma 6.2. $\left(\mathfrak{F}_{ \pm}^{\mathfrak{P}} \times K_{\alpha}\right)^{\text {u.e. }} \models \mathrm{e}^{\mathfrak{D}}\left(\pi_{w_{0}}\right)$ for every infinite $\alpha$.

Proof. Suppose that $W_{ \pm}^{\mathfrak{P}}=\left\{w_{0}, w_{1}, \ldots, w_{b-1}\right\}$, . We put $W_{0}=\left\{w_{0}\right\}$, and $W_{i}=\left\{w_{i}\right\} \times \alpha$. Let $h: W^{\mathfrak{D}, K_{\alpha}} \backslash\left\{w_{0}\right\} \rightarrow \alpha$ be the projection given by the formula $h((w, v))=v$.

First, we prove that $\left(\mathfrak{F}_{ \pm}^{\mathfrak{P}} \times K_{\alpha}\right)^{\text {u.e. }}=\mathrm{e}^{\mathfrak{D}}\left(w_{0}\right)$. To this end we fix an arbitrary non-principal ultrafilter $u$ over $\alpha$, put $X_{i}=\left\{g\left(x_{i}\right)\right\} \times \alpha$ (thus every $X_{i}=W_{j}$ for some j) and for $1 \leq i \leq n$ define ultrafilters $\mu_{i}$ by the following condition

$$
A \in \mu_{i} \Longleftrightarrow h\left(A \cap X_{i}\right) \in u .
$$

Also, put $\mu_{0}=\pi_{w_{0}}$. It is easy to check that $x_{i} R_{\lambda}^{\mathfrak{D}} x_{j}$ implies $\mu_{i}\left(R^{\mathfrak{D}, K_{\alpha}}\right)_{\lambda}^{u . e \cdot} \mu_{j}$, and so $\left(\mathfrak{F}_{ \pm}^{\mathfrak{D}} \times K_{\alpha}\right)^{\text {u.e. }}=\mathbf{k}^{\mathfrak{D}}\left(\mu_{0}, \mu_{1}, \ldots, \mu_{n}\right)$ (for details, see Section 5 of [20]).

Now let us show that for arbitrary ultrafilter $v$, such that $\left\{w_{0}\right\} \notin v\left(\mathfrak{F}_{ \pm}^{\mathfrak{P}} \times K_{\alpha}\right)^{u . e .}=\mathrm{e}^{\mathfrak{D}}(v)$. Notice that $W^{\mathfrak{D}, K_{\alpha}}=\left\{w_{0}\right\} \cup W_{1} \cup \ldots \cup W_{m}$. Hence, by Lemma 2.3 for some $s W_{s} \in v$. Let $u$ be the ultrafilter on $\alpha$ defined by condition

$$
A \in u \Longleftrightarrow h\left(A \cap W_{s}\right) \in u .
$$

But, according to $\left(C\right.$-vi), there exist $w_{k_{1}}, \ldots, w_{k_{n}}$ such that $\mathfrak{F}_{+}^{\mathfrak{P}}=\mathbf{k}^{\mathfrak{D}}\left(p\left(w_{s}\right), w_{k_{1}}, \ldots, w_{k_{n}}\right)$. Now, define ultrafilters $\mu_{i}$ for $i=1, \ldots, n$ by the condition

$$
A \in \mu_{i} \Longleftrightarrow h\left(A \cap W_{k_{i}}\right) \in u .
$$

We claim that $\left(\mathfrak{F}_{ \pm}^{\mathfrak{P}} \times K_{\alpha}\right)^{\text {u.e. }} \models \mathrm{k}^{\mathfrak{D}}\left(v, \mu_{1}, \ldots, \mu_{n}\right)$. Thus $\left(\mathfrak{F}_{ \pm}^{\mathfrak{P}} \times K_{\alpha}\right)^{\text {u.e. }} \models \forall x\left(x \neq w_{0} \rightarrow \mathrm{e}^{\mathfrak{D}}(x)\right)$, and so $\left(\mathfrak{F}_{ \pm}^{\mathfrak{D}} \times K_{\alpha}\right)^{\text {u.e. }}=\mathrm{e}^{\mathfrak{D}}\left(w_{0}\right)$.

Lemma 6.3. Let $u$ be an ultrafilter over $\omega$. Then $\prod_{i \in \omega}^{u}\left(\mathfrak{F}_{ \pm}^{\mathfrak{P}} \times K_{i}\right)$ is isomorphic to $\mathfrak{F}_{ \pm}^{\mathfrak{P}} \times \prod_{i \in \omega}^{u} K_{i}$.

Proof. Let $\left\lceil z_{0}, v_{0}, z_{1}, v_{1}, z_{2}, v_{2}, \ldots\right\rceil=\lceil\bar{z}, \bar{v}\rceil$ be a point of $\prod_{i \in \omega}^{u}\left(\mathfrak{F}_{ \pm}^{\mathfrak{P}} \times K_{i}\right)$. Set $W_{j}=\left\{i \in \omega \mid z_{i}=w_{j}\right\}$ for $j=0, \ldots, m$. By Lemma 2.3 , there exists unique $j$ such that $W_{j} \in u$. Suppose, $W_{j}=\left\{i_{0}, i_{1}, i_{2}, \ldots\right\}$. We put $f\left(\left\lceil z_{0}, v_{0}, z_{1}, v_{1}, z_{2}, v_{2}, \ldots\right\rceil\right)=\left(w_{j},\left\lceil x_{i_{0}}, x_{i_{1}}, x_{i_{2}}, \ldots\right\rceil\right)$. We claim that $f$ is an isomorphism between $\prod_{i \in \omega}^{u}\left(\mathfrak{F}_{ \pm}^{\mathfrak{P}} \times K_{i}\right)$ and $\mathfrak{F}_{ \pm}^{\mathfrak{D}} \times \prod_{i \in \omega}^{u} K_{i}$.

\section{$7 \quad$ Erdös graphs, or putting it all together}

In this section we finally prove the following theorem, the strongest result of this paper.

Theorem 7.1. Let $\mathfrak{D}$ be a minimal connected diagram with inner cycle and let $L=\log \left(\forall x_{0} \mathrm{e}^{\mathfrak{D}}\left(x_{0}\right)\right)$. Then any axiomatisation of $L$ requires infinitely many non-canonical formulas.

In order to do it, we use the following theorem by I. Hodkinson and Y. Venema. Its proof uses probabilistic graphs of Paul Erdös, and we do not reproduce it.

Theorem 7.2 (Theorem 2.3 from [15]). Let $s \geq 2$. There are finite graphs $H_{0}, H_{1}, \ldots$ and surjective homomorphisms $\rho_{i}: H_{i+1} \rightarrow H_{i}$ for $i<\omega$ such that for each $i$,

(E1) for each edge $\{x, y\}$ of $H_{i}$ and each $x^{\prime} \in \rho_{i}^{-1}(x)$, there is $y^{\prime} \in \rho_{i}^{-1}(y)$ such that $\left\{x^{\prime}, y^{\prime}\right\}$ is an edge of $H_{i+1}$,

(E2) $H_{i}$ has no odd cycles of length $\leq i$, 
(E3) $\chi\left(H_{i}\right)=s(\chi$ is the chromatic number).

Relying upon this theorem we show that the condition of Lemma 2.7 indeed holds for axiomatisation $\gamma_{i}^{\mathfrak{D}}$ of the logic in question. Recall that $b$ is the number of points in $\mathfrak{F}_{ \pm}^{\mathfrak{P}}$, and that $W_{ \pm}^{\mathfrak{P}}=$ $\left\{w_{0}, w_{1}, \ldots, w_{b-1}\right\}$.

Given $l$, we announce $n=\left(2^{b l}+1\right) \cdot(b-1)+1$. Then, given $k$, we apply Theorem 7.2 with $s=2^{b k}+1$, and get a sequence of graphs $H_{i}$ and surjective homomorphisms $\rho_{i}: H_{i+1} \rightarrow H_{i}$. Now, we define the sequence $G_{i}$ to be the disjoint union of $H_{i}$ and $K_{2^{b l}+1}$ (here $K_{m}$ is the full graph on $m$ vertices), and extend $\rho_{i}$ to $G_{i+1}$ by putting it identical on $K_{2^{b l}+1}$. Finally, we set $\mathfrak{F}_{i}=\mathfrak{F}_{ \pm}^{\mathfrak{P}} \times G_{i}$, and define morphisms $f_{i}: \mathfrak{F}_{i+1} \rightarrow \mathfrak{F}_{i}$ by $f_{i}\left(w_{0}\right)=w_{0}$ and $f_{i}\left(\left(w_{j}, v\right)\right)=\left(w_{j}, \rho_{i}(v)\right)$ for $j \geq 1$. (E1) guarantees that all $f_{i}$ are indeed p-morphisms. It is easy to see that

$$
\begin{gathered}
\lim _{\leftarrow}\left(\mathfrak{F}_{ \pm}^{\mathfrak{D}} \times G_{i}\right)=\mathfrak{F}_{ \pm}^{\mathfrak{D}} \times \lim _{\leftarrow} G_{i} \text { and } \\
\lim _{\leftarrow}\left(G_{i}\right)=\left(\lim _{\leftarrow} H_{i}\right) \cup K_{2^{b l}+1} .
\end{gathered}
$$

Now we have apply Lemma 5.1 to ensure that (L1), (L2), (L3) hold for formulas $\gamma_{i}^{\mathfrak{D}}$.

(L1): By (E3), $H_{i}$ has chromatic number $2^{b k}+1$, and so it cannot be painted in $2^{b k}$ colours. Since $H_{i}$ is a subgraph of $G_{i}, G_{i}$ also cannot be painted in $2^{b k}$ colours. Thus, by (C1), $\mathfrak{F}_{i} \models \gamma_{k}^{\mathfrak{D}}$.

(L2): By (VII), $K_{2^{b l}+1}$ is a subgraph of $\lim _{\leftarrow} G_{i}$, and so $\lim _{\leftarrow} G_{i}$ cannot be coloured in $2^{b l}$ colours. Again, by (VI) and $(\mathrm{C} 1), \lim _{\leftarrow} \mathfrak{F}_{i}=\gamma_{l}^{\mathfrak{P}}$.

(L3): By (E2), $\lim _{\leftarrow} H_{i}$ is two colourable. Hence $\lim _{\leftarrow}\left(G_{i}\right)$ can be coloured in $2^{b l}+1$ colours, therefore, by $(\mathrm{C} 2), \lim _{\leftarrow} \mathfrak{F}_{i} \not \models \gamma_{n}^{\mathfrak{D}}$ for $n=\left(2^{b l}+1\right) \cdot(b-1)+1$. This finishes the proof of Theorem 7.1.

\section{Main results}

Theorem 8.1. Let $\mathfrak{D}$ be a rooted diagram, all undirected cycles of which pass through its root. Then $(I-i)-(I-x)$ hold.

Proof. It is enough to establish that $\mathrm{e}^{\mathfrak{D}}\left(x_{0}\right)$ is locally modally definable by a generalised Sahlqvist formula, and then use the generalised Sahlqvist theorem [12] on completeness. A rigourous proof of modal definability of $\mathrm{e}^{\mathfrak{D}}\left(x_{0}\right)$ by a generalised Sahlqvist formula can be found in [20], Theorem 4.3. Since the proof is quite long, we do not reproduce it here. A shorter proof of modal definability of $\mathrm{e}^{\mathfrak{D}}\left(x_{0}\right)$ was given earlier in [31].

Theorem 8.2. Let $\mathfrak{D}$ be a minimal rooted diagram with a cycle not passing through its root. Then $(I-i)-(I-i x)$ do not hold for the formula $\mathrm{e}\left(x_{0}\right)=\mathrm{e}^{\mathfrak{P}}\left(x_{0}\right)$ and $\mathcal{C}$, the class of all Kripke frames validating $\forall x_{0} \mathrm{e}\left(x_{0}\right)$.

$(I-i)$ - (I-iii): Since local modal definability implies global definability, it is enough to show that $\forall x_{0} \mathrm{e}\left(x_{0}\right)$ is not globally modally definable. Indeed, $\mathfrak{F}_{ \pm}^{\mathfrak{P}} \times K_{\omega} \not \models \forall x_{0} \mathrm{e}\left(x_{0}\right)$ (Lemma 6.1) but $\left(\mathfrak{F}_{ \pm}^{\mathfrak{P}} \times K_{\omega}\right)^{\text {u.e. }} \models$ $\forall x_{0} \mathrm{e}\left(x_{0}\right)$ (Lemma 6.2), a contradiction to Lemma 2.2.

(I-iv), (I-v), (I-vii), (I-viii): follow immediately from Theorem 7.1.

(I-vi): As it is known (e.g. from [23], but the idea dates back to [26]), to prove that a normal modal $\operatorname{logic} L$ is not axiomatisable with finitely many variables, it is sufficient to construct a sequence of Kripke frames $\mathfrak{F}_{1}, \mathfrak{F}_{2}, \mathfrak{F}_{3}, \ldots$, such that

(a) $\mathfrak{F}_{i} \not \models L$ for all $i$.

(b) for all $k$ there exists $n$ such that $\left(\mathfrak{F}_{n}, \theta\right) \models L$ for every $k$-generated valuation $\theta$ on $\mathfrak{F}_{n}$.

And this can be easily done: take $\mathfrak{F}_{i}=\mathfrak{F}_{ \pm}^{\mathfrak{P}} \times K_{i}$ for all $i \in \omega$. Then (a) follows from Lemma 5.1, (C2) and (b) follows from Lemma 5.1, (C1), and the fact that $K_{n}$ cannot be painted in less then $n$ colours (take $\left.n=2^{b k}+1\right)$.

$(I-i x)$ : it is clear that $\mathfrak{F}_{ \pm}^{\mathfrak{P}} \times K_{\omega} \notin \mathcal{C}$ (Lemma 6.1), but $\mathfrak{F}_{ \pm}^{\mathfrak{P}} \times K_{\omega}=L$ (Lemma 5.1, (C1)).

$(I-x)$ : suppose that there exists a first-order formula $\gamma$ such that $\mathfrak{F}=\gamma$ iff $\mathfrak{F}=L$ for each Kripke frame $\mathfrak{F}$. Then by Lemma 5.1, (C2) for all $i<\omega \mathfrak{F}_{ \pm}^{\mathfrak{P}} \times K_{i} \models \neg \gamma$, but by Lemma $6.3, \prod_{i \in \omega}^{u}\left(\mathfrak{F}_{ \pm}^{\mathfrak{P}} \times K_{i}\right)=$ $\mathfrak{F}_{ \pm}^{\mathfrak{P}} \times\left(\prod_{i \in \omega}^{u} K_{i}\right)$, therefore $\prod_{i \in \omega}^{u}\left(\mathfrak{F}_{ \pm}^{\mathfrak{P}} \times K_{i}\right) \models \gamma$, since $\prod_{i \in \omega}^{u} K_{i}$ is isomorphic to $K_{\alpha}$ for some infinite $\alpha$ (cf. the proof of Theorem 10 of [16]). This contradicts Proposition 2.4. 


\section{Discussion}

Let us discuss the family of Kripke frame classes that are covered by our theorem. They are defined by first-order formulas of the form $\forall x_{0} \exists x_{1} \ldots \exists x_{n} \wedge x_{i} R_{\lambda} x_{j}$. This family is chosen because it is large enough to generate modal logics of both types of the dichotomy, and narrow enough to allow the dichotomy to be proven. How interesting is this family? On the one hand, these formulas may seem rather artificial, since very few of them may be said to be orthodox in modal logic, though they include well known reflexivity and reflexive-successor conditions. It also seems difficult to invent a practical reasoning problem involving these formulas. On the other hand, if we omit the universal quantifier $\forall x_{0}$, then we obtain existential conjunctive formulas, which recently have received much attention both in the logical and computer science communities under the name of conjunctive queries. If we close the class of existential conjunctive formulas with many free variables under restricted universal quantification, we obtain the class of $\forall \exists$-formulas discussed in Section 6 of [20], which includes many more first-order conditions traditional to modal logic. Thus the formulas from this paper may be understood as 'building blocks' for more complicated and interesting formulas, and so our result can be considered as a step towards more general dichotomy theorems. First-order formulas of the form $\mathrm{a}(x)=\exists y\left(x R_{\lambda} y \wedge \mathrm{b}(y)\right)$ where $\mathrm{b}(y)$ is a generalised Kracht formula may be good candidates for further research; see [1] and [11] for known information about the corresponding modal logics. However, it is still not clear how far this dichotomy can be pushed. It is also interesting if the condition

$(I-x i)\{\mathfrak{F}|\mathfrak{F}|=\log (\mathcal{C})\}$ is $\Delta$-elementary

may be added to $(I-i)-(I-x)$ without breaking the dichotomy (cf. [2]).

Acknowledgements. The author thanks Philippe Balbiani and Ian Hodkinson for helpful and fruitful discussions. This research was supported by RFBR - CNRS grant 11-01-93107. The preparation of the final version of the paper was supported by RFBR - CNRS grant 14-01-93105.

\section{References}

[1] P. Balbiani, I. Shapirovsky, and V. Shehtman. Every world can see a Sahlqvist world. In Advances in Modal Logic 6, pages 69-85, 2006.

[2] J. van Benthem. Modal formulas are either elementary or not $\Sigma \Delta$-elementary. J. Symb. Log., 41(2):436-438, 1976.

[3] P. Blackburn, M. de Rijke, and Y. Venema. Modal Logic. Cambridge University Press, 2002.

[4] J. Bulian. Exploring canonical axiomatisations of representable cylindric algebras, final year project at Imperial College London, Department of Computing, 2011.

[5] J. Bulian, I. Hodkinson. Bare canonicity of representable cylindric and polyadic algebras. Annals of Pure and Applied Logic, 164(9):884-906, 2013.

[6] A. Chagrov and L. Chagrova. The truth about algorithmic problems in correspondence theory. In Advances in Modal Logic 6, pages 121-138, 2006.

[7] L. Chagrova. An undecidable problem in correspondence theory. J. Symb. Log., 56(4):1261-1272, 1991.

[8] D. Gabbay, A. Kurucz, F. Wolter, and M. Zakharyaschev. Many-dimensional modal logics: theory and applications. Studies in Logic and the Foundations of Mathematics, 148. Elsevier, 2003.

[9] D. Gabbay and V. Shehtman. Products of modal logics, part 1. Journal of the IGPL, 6:73-146, 1998.

[10] R. Goldblatt. Mathematics of modality. Lecture notes, vol. 43, CSLI Publications, Standford, CA, 1993. 
[11] R. Goldblatt and I. Hodkinson. The McKinsey-Lemmon logic is barely canonical. The Australasian Journal of Logic, 5:1-19, 2007.

[12] V. Goranko and D. Vakarelov. Elementary canonical formulae: extending Sahlqvist's theorem. Annals of Pure and Applied Logic, 141(1-2):180-217, 2006.

[13] E. Hemaspaandra and H. Schnoor. On the complexity of elementary modal logics. In Symposium on Theoretical Aspects of Computer Science, pages 349-360, 2008.

[14] I. Hodkinson. Hybrid formulas and elementarily generated modal logics. Notre Dame Journal of Formal Logic, 47(4):443-478, 2006.

[15] I. Hodkinson and Y. Venema. Canonical varieties with no canonical axiomatisation. Trans. Amer. Math. Soc, 357:4579-4605, 2003.

[16] G. Hughes. Every world can see a reflexive world. Studia Logica - An International Journal for Symbolic Logic, 49:175-181, 1990.

[17] H. Keisler and C. Chang. Model Theory, 3rd ed. Elsevier Science publishers, The Netherlands, 1990.

[18] S. Kikot. An extension of Kracht's theorem to generalized Sahlqvist formulas. Journal of Applied Non-Classical Logic, 19/2:227-251, 2009.

[19] S. Kikot. On modal definability of Horn formulas. In Topology, Algebra and Categories in Logic, pages 175-178. Marseille, 2011.

[20] S. Kikot and E. Zolin. Modal definability of first-order formulas with free variables and query answering. In Journal of Applied Logic, 11:190-216, 2013.

[21] Kracht, M.: How completeness and correspondence theory got married. In: M. de Rijke (Ed.), Diamonds and Defaults, pp. 175-214. Synthese Library, Kluwer (1993)

[22] Kracht, M.: Tools and Techniques in Modal Logic. Studies in Logic and the Foundations of Mathematics, 142. Elsevier (1999)

[23] A. Kurucz. On axiomatising products of Kripke frames, part II. In C. Areces and R. Goldblatt, editors, Advances in Modal Logic 7, pages 219-230. King's College Publications, 2008.

[24] A. Kurucz. On the complexity of modal axiomatisations over many-dimensional structures. In Advances in Modal Logic 8, pages 256-270, 2010.

[25] A. Kurucz, F. Wolter, and M. Zakharyaschev. Islands of tractability for relational constraints: Towards dichotomy results for the description logic EL. In Advances in Modal Logic 8, pages 271$291,2010$.

[26] L. Maksimova, V. Shehtman, and D. Skvortsov. The impossibility of a finite axiomatization of Medvedevs logic of finitary problems. In Soviet Math. Dokl, 20:394-398, 1979.

[27] J. Michaliszyn and J. Otop. Decidable elementary modal logics. In LICS, pages 491-500, 2012.

[28] D. Vakarelov. Modal definability in languages with a finite number of propositional variables and a new extension of the Sahlqvist's class. In Advances in Modal Logic 4, pages 499-518, 2002.

[29] D. Vakarelov. Extended Sahlqvist formulae and solving equations in modal algebras. In 12-th International Congress of Logic Methodology and Philosophy of Science, August 7-13. Abstracts, page 33. Oviedo, Spain, 2003.

[30] Y. Venema. Canonical pseudo-correspondence. In Advances in Modal Logic 2, pages 421-430, 1998.

[31] E. Zolin. Query answering based on modal correspondence theory. In Proceedings of the 4th "Methods for modalities" Workshop (M4M-4), pages 21-37, 2005. 